\title{
Syntaxonomy of chalk outcrop vegetation of the order Thymo cretacei-Hyssopetalia cretacei
}

\author{
Yakiv Didukh ${ }^{1}$, Olga Chusova ${ }^{1, *} \&$ Olga Demina ${ }^{2}$
}

Keywords: Central Russian Upland, Hyssopus flora, lowered alpine plants classification.

Ključne besede: Srednjerusko višavje, flora rodu Hyssopus, klasifikacija nizkih alpinskih rastlin.

Received: 14. 3. 2017

Revision received: 14. 7. 2017

Accepted: 10. 11. 2017

\section{Co-ordinating Editor: \\ Orsolya Valkó}

\begin{abstract}
The order Thymo cretacei-Hyssopetalia cretacei Didukh 1989 combines chalk outcrop plant communities of the southwestern Central Russian Upland. Its specificity can be attributed to a rather peculiar and heterogeneous flora with a large number of endemic species. The question about its origin has caused a lively discussion, which has been going on since the late nineteenth century. Since 1989 works on the classification of these communities have frequently been carried out, but until today no unanimous decision could be reached. The purpose of our research was to conduct a critical analysis of the syntaxonomical structure of Cretaceous outcrop vegetation and to show its difference from the steppe vegetation of the class Festuco-Brometea Br.-Bl. et Tx. ex Soó 1947. The territory of our research covers the southwestern foothills of the Central Russian Upland and the Donetsk Range, located only within the steppe zone and characterised by Cenomanian chalk outcrops. In total 354 relevés were used for the analysis. The modified TWINSPAN classification was used for the analysis. Our research has shown that the order Thymo cretacei-Hyssopetalia cretace includes twelve associations belonging to three alliances: Artemisio hololeucae-Hyssopion cretacei Romashchenko et al. 1996, Euphorbio cretophilae-Thymion cretacei Didukh 1989 and Centaureo carbonatae-Koelerion talievii Romashchenko et al. 1996.

Izvleček

V red Thymo cretacei-Hyssopetalia cretacei Didukh 1989 združujemo rastlinske združbe, ki jih najdemo na krednih izdankih v jugozahodnem Srednjeruskem višavju. Njegova specifičnost je posledica posebne in heterogene flore z velikim številom endemitov. Njen izvor je povzročil živahno diskusijo, ki traja že od konca 19. stoletja. Številne raziskave o klasifikaciji teh združb so izvajali od leta 1989, vendar do danes ni prišlo do soglasnega pojasnila. Namen naše raziskave je narediti kritično sintaksonomsko analizo vegetacije krednih izdankov in prikazati razlike od stepske vegetacije razreda Festuco-Brometea Br.-Bl. et Tx. ex Soó 1947. Območje raziskav obsega jugozahodna vznožja Srednjeruskega višavja in gorske verige Doneck, ki se nahaja znotraj stepske cone na cenomanijskih krednih izdankih. $\mathrm{V}$ analizi smo uporabili 354 vegetacijskih popisov in modificirano TWINSPAN klasifikacijsko metodo. Pokazali smo, da v red Thymo cretacei-Hyssopetalia cretacei uvrščamo 12 asociacij, ki pripadajo trem zvezam: Artemisio hololeucae-Hyssopion cretacei Romashchenko et al. 1996, Euphorbio cretophilae-Thymion cretacei Didukh 1989 in Centaureo carbonatae-Koelerion talievii Romashchenko et al. 1996.
\end{abstract}

1 M.G. Kholodny Institute of Botany, National Academy of Sciences of Ukraine, 2 Tereschenkivska St., 01601 Kyiv, Ukraine. E-mail: olgachusova@gmail.com

2 Karachay-Circassian State University, 29 Lenina St., 369200 Karachaevsk, Russia. E-mail: ondemina@yandex.ru

* Corresponding author 


\section{Introduction}

The specificity of chalk outcrop flora and vegetation has been noted by botanists since the late nineteenth century and caused a heated debate about its origins (Tsinger 1885; Litvinov 1891, 1902, Taliev 1897, 1904, 1905, Pachos'kyy 1910, Kozo-Polyansky 1910, 1925, 1931, Szafer 1928, Kleopov 1930, Lavrenko 1932, Grosset 1935, Hryn' 1938). While Taliev (1905) regarded cretaceous flora as young and associated with human activities, most scientists accept the theory according to which its main core is relict and had formed long before the ice age. Because of the large number of endemic species confined to Cretaceous outcrops, this flora was called "Hyssopus flora" (after its typical element Hyssopus cretaceus Dubj) (Kozo-Polyansky 1931). Also, Kozo-Polyansky showed that the flora of Cretaceous outcrops is heterogeneous and includes another component besides "Hyssopus flora", which he called "lowered alpine plants" (the unit of periglacial Pleistocene steppe). Typical representatives of this complex are Carex pediformis C. A. Mey., Carex humilis Leyss., Clausia aprica (Stephan) Trotsky, Alyssum lenense Adams and others. The core of this flora is formed by specific xerophytic endemic surface plants and squat plants confined to dry chalky substrates. They are characterised by grey stellate and weblike pubescence or waxy coating (Hyssopus cretaceus, Linaria cretacea Spreng., Scrophularia cretacea Spreng., Silene cretacea Spreng), which shows the processes of speciation in such conditions. Typical components of the flora are mountainous Mediterranean species, whose nearest occurence is in the Crimean Mountains (Euphorbia petrophila C. A. Mey., Asperula tephrocarpa (incl. A. cretacea, A. supina), Silene supina M. Bieb., Polygala mediterranea, Hedysarum ucrainicum (incl. H. tauricum)), and species of broader South European distribution (Helianthemum canum s.l. (L.) Hornem., Jurinea stoechadifolia (M. Bieb.) DC., Pimpinella tragium ssp. titanophylla (Woronow) Tutin, Linum czernjaevii Klok., Taraxacum serotinum (Waldst. \& Kit.) Fisch., Scutellaria supina L.). On the other hand, there are Pontic-Siberian steppe species (Artemisia salsoloides Willd., Psephellus marschallianus (Spreng.) K. Koch, Gypsophila altissima (incl. G. oligosperma), Cephalaria uralensis (Murray) Roem. \& Schult., Astragalus albicaulis DC., Clausia aprica (Stephan) Korn.-Trotzky, Hedysarum grandiflorum Pall., Alyssum lenense Adams, Androsace villosa (incl. A. kozopoljanskyi), Polygala sibirica L.) and even Central Asian species (Atrophaxis frutescens (L.) K. Koch, Artemisia salsoloides, Krascheninnikovia ceratoides (L.) Gueldenst.). Thus, the floristic composition of Cretaceous outcrops is rather peculiar and heterogeneous, and it vastly differs from zonal steppe vegetation. As for taxonomy, it is characterised by the presence of endemic races, which sometimes get species status (Dobrochayeva et al.1987, Klokov \& Dobrochayeva 1974, Cvelev 1996).

The vegetation classification of the dominant basis is reflected in the works of Semenova-Tian-Shanskaya (1954), Golitsyn et al. (1963), Golitsyn (1965), Hryn' (1973), Kondratyuk et al. (1988), Shelyag-Sosonko et al. (1991), Tkachenko et al. (1998) and Vakarenko \& Dubyna (2006). It was found that the main dominant species of these groups are Hyssopus cretaceous, Thymus calcareus, Asperula tephrocarpa, Scrophularia cretacea, Pimpinella tragium ssp. titanophylla, Artemisia hololeuca, A. salsoloides, Plantago maritima ssp. ciliata Printz, Jurinea stoechadifolia, Krascheninnikovia ceratoides, Silene cretacea and Festuca cretacea T. I. Popov \& Proskor., which form together a variety of combinations. Cretaceous outcrop vegetation is quite different from zonal steppe vegetation (Didukh 1996). Based on the specific conditions of substrate formation and apparent characteristics, H. Vysots'kyy (1915), B. Kozo-Polyanskyi (1931) and E. Lavrenko (1961) named it "thymyannyky" (after the genus name Thymus). Ya. Didukh (1981) considered it an analogue of Mediterranean tomillares.

The first floristic classification of Cretaceous vegetation was obtained by Ya. Didukh (1989). It was attributed to the order Thymo cretacei-Hyssopetalia cretacei Didukh 1989 and considered as part of the class Festuco-Brometea. Later, in 1996 and in virtue of additional studies, the chalky outcrop communities were assigned to the new class Helianthemo-Thymetea Romashchenko et al. 1996, in which there are three alliances: Artemisio hololeucae-Hyssopion cretacei, Euphorbio cretophilaeThymion cretacei and Centaureo carbonatae-Koelerion talievii. After that studies have been conducted both in Ukraine (Didukh \& Korotchenko 1996) and in Russia (Averinova 2005, 2011, 2012, 2014, Demina, 2011, 2012, 2014, 2016; Poluyanov 2009, 2012, Sereda 2003, $2008,2009)$ that made some alterations to this classification. In the later construction EuroVegChecklist (Mucina et al. 2016), the order Hyssopetalia cretacei was included in the class Festuco-Brometea. Purpose of our research was to conduct a critical analysis of the syntaxonomical structure of Cretaceous outcrop vegetation and to show its difference from the steppe vegetation of the class Festuco-Brometea.

Our key task was to critically analyse the classification of syntaxa described in previous years and to add novel information based on new data representing the whole research area within Ukraine and the Russian Federation. 


\section{Description of the research territory}

The Central Russian Upland extends from Northwest to Southeast from the right bank of the Oka River to the Donetsk Range. In the West it is adjacent to the Dnieper Lowland and in the East to the Oka-Don Lowland; in the North its frontier follows the watershed of the rivers Desna, Oka and Don, and in the South it follows the watershed of the rivers Dnieper, Siversky Donets and Don (Karandeeva 1957). The foothills of the Central Russian Upland are the plane inclined to the South and Southwest with elevations of about 190-200 m. It is deeply dissected by draws and ravines. In the South-East it borders with the Donetsk Range and the Siversky Donets valley. The Donetsk Range can be considered as foothills of the Central Russian Upland. It is located in the Donetsk region and the South of the Lugansk region, which belong to Ukraine and to the Rostov region of the Russian Federation. In the Donetsk Range, cretaceous outcrops appear only in the valleys of Siversky Donets and some of its feeders.

The south-western foothills of the Central Russian Upland are located within the forest-steppe and steppe zones and cover the territory of the Kharkiv, Donetsk and Luhansk regions of Ukraine and Kursk and the Voronezh, Belgorod and Rostov regions of the Russian Federation (Baranov 1969). The research territory, $50^{\circ} 52^{\prime} 21^{\prime \prime} \mathrm{N}$, $38^{\circ} 4^{\prime} 26^{\prime \prime} \mathrm{E}$ to $49^{\circ} 30^{\prime} 44^{\prime \prime} \mathrm{N}, 36^{\circ} 41^{\prime} 48^{\prime \prime} \mathrm{E}$ and $49^{\circ} 30^{\prime}$ $53^{\prime \prime} \mathrm{N}, 43^{\circ} 26^{\prime} 04^{\prime \prime} \mathrm{E}$ to $47^{\circ} 27^{\prime} 31^{\prime \prime} \mathrm{N}, 40^{\circ} 27^{\prime} 30^{\prime \prime} \mathrm{E}$, covers the southwestern foothills of the Central Russian Upland and the Donetsk Range. It comprises only the steppe zone and is characterised by Cenomanian chalk outcrops. To the North, Cretaceous outcrops occur within the forest-steppe zone, featuring hyper-carbonatophiles (Thymus calcareus Klokov \& Des.-Shost., Inula ensifolia L., Polygala sibirica, Bupleurum falcatum L.), but in spe- cies composition and structure, these communities are very different from Hyssopetalia cretacei (Golitsyin 1936, Honcharenko 2000).

The recent terrain of the Central Russian Upland is a result of the Neogene-Quaternary neotectonic surface emergence on the height of 200 meters, which was accompanied by tectonic and erosion-accumulative deformation. Tectonic deformations are the result of structural heterogeneity of submerging basal complex, the presence of deep lateral and transverse crust fractures and inherited geological structure of the platform's sedimentary sheath.

River valleys of the Siversky Donets and its feeders (Krasna, Aidar, Oskol) are wide, with steep right banks, which can be up to $100 \mathrm{~m}$ high. The right banks are dissected by a system of ravines and gullies. The ravines are fairly long for the most part and sometimes have the shape of a "circus". These formations indicate intense erosion processes in the past and present. The left banks are gently sloping, with well-developed upland terraces.

The research area is under conditions of sub-continental (subarid) climate, characterised by large temperature amplitudes between winter and summer periods. The average annual temperature is $7-8{ }^{\circ} \mathrm{C}$, the average January temperature ranges from -4 to $-6{ }^{\circ} \mathrm{C}$, the average July temperature from +21 to $+24^{\circ} \mathrm{C}$. Annual precipitation is in the range of $450-500 \mathrm{~mm}$ in most parts of the research territory, ranging up to $550 \mathrm{~mm}$ in the northwestern part in the Russian Federation (Figure 1a). The central part of the reseach territory is characterised by considerable continentality and longer droughts in the summer period (Figure 1b). The most continental and most arid territory is the south-eastern part of the Donetsk Range (Figure 1c) with fairly irregular precipitation of 400-420 $\mathrm{mm}$ per year. The largest is the spring and early summer rainfall, followed by a sharp decline in the second half of summer and a subsequent rise in winter (Hrytsenko 2004, Weather in Ukraine, 2014).
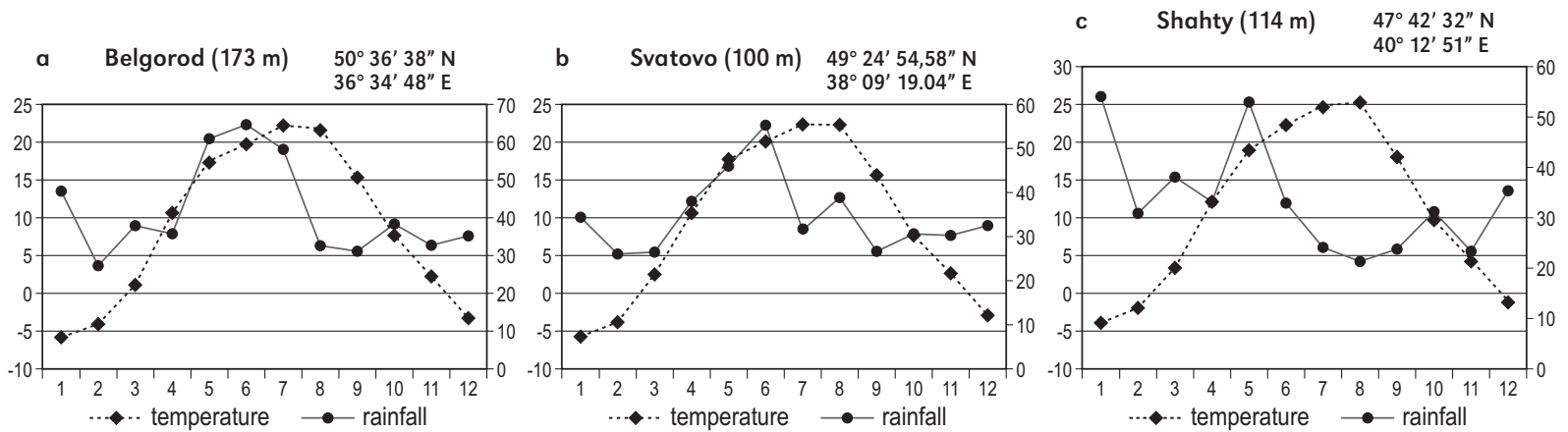

Figure 1: Walter climographs based on climatic data from Hrytsenko (2004) and Weather in Ukraine (2014) for the northern (a), central (b) and southern (c) part of the research territory.

Slika 1: Walterjevi klimadiagrami na osnovi podatkov iz Hrytsenko (2004) in Weather in Ukraine (2014) za severni (a), srednji (b) in južni (c) del raziskovanega območja. 
As the Walter climograph shows, such distribution determines a rather long (4-5 months) dry season, and its effect is further enhanced on the dry southern chalk slopes. This is one of the factors that determine the specificity of flora and vegetation. Under such conditions, black soils are formed in upland areas and rendzinas (sodcalcareous soils) and regosols (deluvial-carbonate soils) on the slopes with the chalk outcrops.

\section{Materials and Methods}

For the analysis we used 354 relevés made by Ya. Didukh and O. Chusova in the Kharkiv, Donetsk and Lugansk region of Ukraine and by O. Demina in the Rostov region of the Russian Federation from 1987 to 2015. In addition, we used 51 relevés from the Kharkiv region kindly provided by O. Bezrodnova as well as 178 relevés published by K. Romashchenko et al. (1996), A. Poluyanov (2009, 2012), E. Averinova (2014) and M. Sereda (2008, 2009). To estimate the situation of Cretaceous outcrop vegetation in relation to other types, we used about 300 relevés of xerophytic herbaceous vegetation from the same territory. The plot sizes for relevés were from 25 to $100 \mathrm{~m}^{2}$. Unpublished releves were compiled in a TURBOVEG (Hennekens \& Schaminee 2001) database. Unpublished relevés sampled by Chusova (112 relevés) were added to the GIVD World Warehouse in the Ukrainian Grassland Database (EU-UA-001). Unpublished releves sampled by Didukh (242 relevés) were added to the Vegetation of Lugansk region, Ukraine (EU-UA-008).

Floristic data were processed by using JUICE (Tichý 2002). Clusterisation and syntaxa extraction were performed using a modified TWINSPAN algorithm (minimum number of groups - 2, pseudospecies cut level - 05 25; Simpson coefficient) (Roleček et al. 2009) implemented in the software package JUICE (Tichý 2002). We also used the method of cluster analysis included in the STATISTICA 6.0 software package.

The diagnostic species of clusters were determined by the phi coefficient of fidelity (Chytrý et al. 2002). Using Fisher's exact test at $\alpha=0.05$, species with a value of phi $\geq 0.3$ were considered diagnostic, species with a value of phi $\geq 0.5$ highly diagnostic. For dominant species, we adopted a projective coverage threshold of $>25 \%$ in the herbage and a $10 \%$ threshold of frequency.

We identified selected syntaxa on the basis of a critical analysis of publications (Didukh 1989, Romashchenko et al. 1996, Didukh \& Korotchenko 1998, Sereda 2008, 2009, Poluyanov 2009, 2012, Demina 2011, Averinova 2014, Mucina et al. 2016). For the JUICE analysis, we have used the associations' typical relevés to identify them.
Nomenclature of syntaxa follows the International Code of Phytosociological Nomenclature (Weber et al. 2000); nomenclature of taxa is in accordance with the Euro+Med Plant base service (2006) or, in case of taxa not listed there, Flora Europaea (Tutin 1968, 1972, 1976, 1980, 1993). We did use the name Hyssopus cretaceous Dubj. instead of Hyssopus officinalis L., though. Since the species of chalk outcrops are rather specific and narrowly local, many of them are endemic. However, in the Flora Europaea, these species are only listed as synonyms. Usually we have reduced them to synonyms that are accepted for Europe, but as for Hyssopus cretaceus, the situation is different. Within the research territory there are two subspecies of Hyssopus officinalis, which both form associations of petrophytic type, but are quite different in terms of morphology and ecology. Hyssopus cretaceus is reduced in Flora Europaea and Euro+Med Plant to the synonym Hyssopus officinalis ssp. montanus (Jord. \& Fourr.) Briq., which occurs only on the chalk substrate in communities of the order Thymo cretacei-Hyssopetalia cretacei. Another subspecies is sometimes found on sandy soils (Demina 2012). Since Hyssopus officinalis ssp. montanus plays a very important role in the communities we are considering and in order to separate it from subspecies with a different ecology, we decided to use the synonym here.

In the species composition analysis, it was taken into account that in our study region many species typical for Ukraine and southern regions are replaced by species that are similar in terms of environmental characteristics (e.g. Euphorbia cretophila Klokov and E. petrophila, Jurinea brachycephala Klokov and J. stoechadifolia, Genista tanaitica P. A. Smirn. and G. tinctoria L.).

\section{Results and Discussion}

Most communities of Cretaceous outcrops are characterised by small species variety and high diversity of coenoses. The presence of a large number of local endemics among the dominant species is also characteristic for them. Some of the dominant species have diagnostic value at the level of associations, but the most typical species of these groups (Thymus calcareus, Pimpinella tragium (Woronow) Tutin, Artemisia hololeuca, Hyssopus cretaceus) are characterised by a fairly wide range. These features are important when selecting syntaxa.

Different authors in various regions described four alliances (Didukh 1989, Romashchenko et al. 1996, Averinova 2014) and 21 associations (Didukh 1989, Romashchenko et al. 1996, Didukh \& Korotchenko 1997, Sereda 2009, Poluyanov 2009, 2012, Demina 2011, 2012, Averinova 2014). We conducted a critical analysis whereby it was established that the class Helianthemo- 
Tymetea is represented by 14 associations within the territory of the south-western foothills of the Central Russian Upland. A tree diagram was built based on the assessment of species similarity. There are two clusters in this diagram (Figure 2). Cluster "A" covers communities of petrophy-

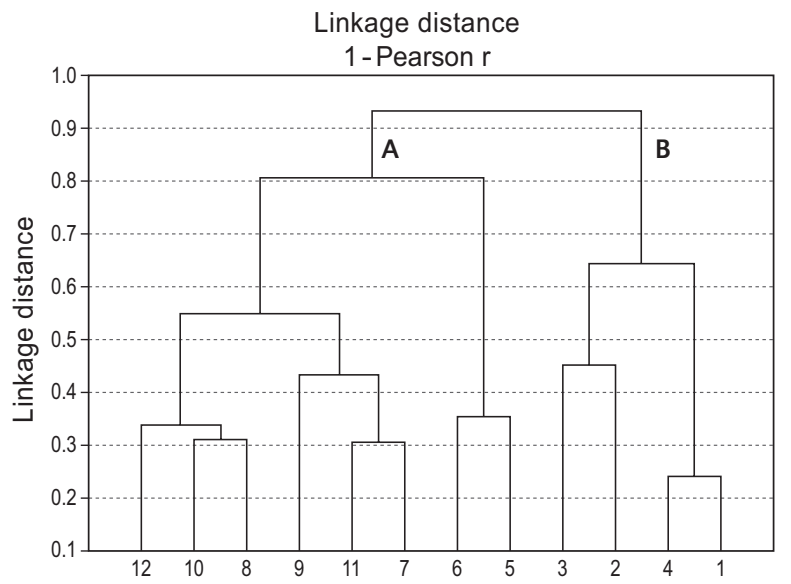

tous steppes ("lowered alpine plants") and falls into the two alliances Centaureo carbonatae-Koelerion talievii and Euphorbio cretophilae-Thymion cretacei. Cluster "B" corresponds to the alliance Artemisio hololeucae-Hyssopion cretacei and covers typical tomillares.
Figure 2: Tree diagram of chalk outcrop vegetation communities distribution (syntaxa numbering shown in Table 1). A - lowered alpine plants (Centaureo carbonatae-Koelerion talievii and Euphorbio cretophilae-Thymion cretacei); B -typical tomillares (Artemisio hololeucae-Hyssopion cretacei).

Slika 2: Dendrogram vegetacije krednih izdankov (oštevilčenje sintaksonov kot v Tabeli 1). A - nizke alpinske rastline (Centaureo carbonatae-Koelerion talievii in Euphorbio cretophilae-Thymion cretacei); B - tipični tomiljar (Artemisio hololeucae-Hyssopion cretacei).

Table 1: Synoptic table of chalk outcrop vegetation communities with percentage frequencies.

Tabela 1: Sinoptična tabela rastlinskih združb krednih izdankov s frekvencami v odstotkih.

\begin{tabular}{lcccccccccccc}
\hline Consecutive number & 1 & 2 & 3 & 4 & 5 & 6 & 7 & 8 & 9 & 10 & 11 & 12 \\
& 15 & 12 & 20 & 107 & 29 & 19 & 24 & 14 & 15 & 26 & 15 & 46 \\
\hline
\end{tabular}

Species richness

D. s. for ass. Artemisio nutantis-Plantaginetum salsae

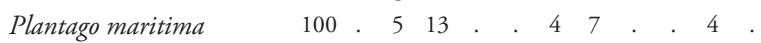

$\begin{array}{llllllllllllllll}\text { Artemisia nutans } & 80 & 8 & . & 3 & . & 4 & 7 & . & . & .\end{array}$

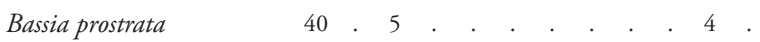

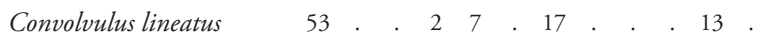

D. s. for ass. Erysimo cretacei-Festucetum cretacei

Erysimum ucrainicum

Melilotus officinalis

Festuca cretaced

. 585

. $75 \cdot 10$

20100408

D. s. for ass. Lepidio meyeri-Scrophularietum cretacei

Lepidium meyeri

$\begin{array}{cccccccccccc}. & . & 45 & . & . & . & . & . & . & . & . & . \\ 13 & . & 69 & . & . & . & 8 & . & . & . & 8 & . \\ 7 & 33 & 85 & 3 & 3 & . & 33 & 21 & . & . & 29 & . \\ . & . & 30 & . & . & . & 4 & . & . & . & . & . \\ 13 & 42 & 30 & 10 & 10 & . & 17 & . & 13 & 20 & 4 & 20 \\ 7 & 33 & 30 & 11 & 28 & . & 17 & 21 & 40 & . & 25 & .\end{array}$

Hedysarum cretaceum

Galium octonarium

Artemisia absinthium

Poa compressa

$\begin{array}{llllllllllll}7 & 33 & 30 & 11 & 28 & \text {. } & 17 & 21 & 40 & \text {. } & 25\end{array}$.

D. s. for ass. Artemisio hololeucae-Polygaletum cretaceae

$\begin{array}{llllllllllll}\text { Artemisia hololeuca } & 73 & 1 & 6 & 64 & 3 & 29 & 21 & 20 & 16 & \text {. }\end{array}$

D. s. for ass. Euphorbio cretophilae-Jurinetum brachycephalae

Pinus sylvestris

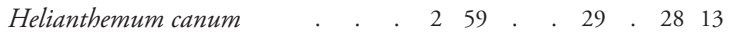

D. s. for ass. Genisto scythicae-Artemisietum salsoloides

Genista scythica

Rhaponticoides taliewii

Leontodon biscutellifolius

Linum tenuifolium

Odontarrhena tortuosa

Linaria vulgaris

\begin{tabular}{lcccccccccccc} 
Consecutive number & 1 & 2 & 3 & 4 & 5 & 6 & 7 & 8 & 9 & 10 & 11 & 12 \\
& 15 & 12 & 20 & 107 & 29 & 19 & 24 & 14 & 15 & 26 & 15 & 46 \\
\hline
\end{tabular}

Diplotaxis cretacea

Polygala comosa

Linum hirsutum

D. s. for ass. Hedysaro grandiflori-Centauretum sumensis

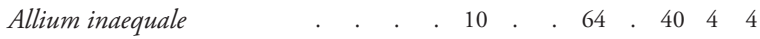

Hedysarum grandiflorum . . . . $\begin{array}{llllllllll}7 & 31 & 42 & 13 & 100 & 4 & 4 & 4\end{array}$.

Thymelaea passerina

Asperula cynanchica

Adonis vernalis

Seseli annuum

Pimpinella saxifraga

Phlomis herba-venti

Salvia verticillata

Aster amellus

Asparagus officinalis

Caragana frutex

Centaurea orientalis

Echinops ritro

. . . . . . 43 .

$\begin{array}{lllllllllll}. & . & 3 & 3 & 53 & 4 & 93 & . & 4 & 4 & 48\end{array}$

D. s. for ass. Androsacio koso-poljanskii-Caricetum humilis

Androsace villosa subsp.

koso-poljanskii

$10012 \quad 8 \quad 57$

D. s. for ass. Bupleuro falcatae-Stipetum capillatae

$\begin{array}{lllllllllllll}\text { Bupleurum falcatum } & 13 & \text {. } 10 & 23 & 45 & \text {. } 21 & 86 & 20 & 76 & 42 & 59\end{array}$

Anisantha tectorum

Salvia nemorosa

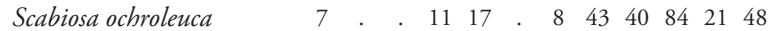

D. s. for ass. Gypsophilo oligospermae-Campanuletum sibiricae

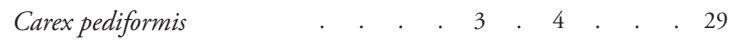

$\begin{array}{lllllllllll}\text { Koeleria pyramidata } & . & 8 & 20 & 3 & 24 & . & 4 & & 16 & 71 .\end{array}$ 


\begin{tabular}{|c|c|c|c|c|c|c|c|c|c|c|c|c|}
\hline Consecutive number & $\begin{array}{c}1 \\
15\end{array}$ & $\begin{array}{c}2 \\
12\end{array}$ & $\begin{array}{c}3 \\
20\end{array}$ & $\begin{array}{c}4 \\
107\end{array}$ & $\begin{array}{c}5 \\
29\end{array}$ & $\begin{array}{c}6 \\
19\end{array}$ & $\begin{array}{c}7 \\
24\end{array}$ & $\begin{array}{c}8 \\
14\end{array}$ & $\begin{array}{c}9 \\
15\end{array}$ & $\begin{array}{l}10 \\
26\end{array}$ & $\begin{array}{l}11 \\
15\end{array}$ & \\
\hline Nonea pulla & $\cdot$ & . & . & . & . & . & 4 & 14 & 7 & . & 33 & \\
\hline Bromopsis riparia & . & - & . & 6 & 3 & 11 & 17 & 57 & 33 & 12 & 75 & \\
\hline Salvia nutans & . & . & . & 1 & 38 & 26 & 25 & 50 & 27 & 52 & 83 & \\
\hline Galatella villosa & . & . & $\cdot$ & . & . & . & . & . & . & . & 38 & \\
\hline Veronica spicata & . & . & . & 3 & 3 & . & 21 & 14 & . & 8 & 29 & \\
\hline Inula aspera & . & . & 5 & 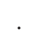 & · & . & 8 & . & 7 & . & 21 & \\
\hline \multicolumn{13}{|c|}{ D. s. for ass. Carici humilis-Thymetum calcarei } \\
\hline Astragalus austriacus & 7 & . & $\cdot$ & . & . & . & . & 43 & 33 & 4 & 29 & \\
\hline Thalictrum minus & $\cdot$ & . & $\cdot$ & $\cdot$ & 24 & 42 & 29 & 43 & . & 36 & 67 & \\
\hline Oxytropis pilosa & . & . & . & 2 & . & . & 4 & . & 7 & . & 17 & \\
\hline Jurinea arachnoidea & 7 &. & . & 4 & 3 & . & 42 & 29 & 20 & 4 & 46 & \\
\hline Viola rupestris & . & . & $\cdot$ & . & . & . & . & . & . & 4 & $\cdot$ & \\
\hline $\begin{array}{l}\text { Helianthemum nummu- } \\
\text { larium }\end{array}$ &. & . & . & . & . & . & . & 36 & . & 4 & . & \\
\hline Asperula tinctoria & . & . & . & 2 & . & . & . & . & . & . & 4 & \\
\hline Anthericum ramosum & $\cdot$ & . & $\cdot$ & . & 21 & . & . & 21 & 7 & 8 & . & \\
\hline Linum perenne & . & . & . & . & . & . & . & 14 & . & . & . & \\
\hline Echium vulgare & . & . & 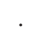 & $\cdot$ & $\cdot$ & . & 4 & . & . & 4 & . & \\
\hline Vincetoxicum hirundinaria & . & . & 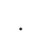 & . & 3 & . & . & . & 7 & 36 & 8 & \\
\hline Pilosella officinarum & . & . & . & $\cdot$ & $\cdot$ & . & $\cdot$ & . & 7 & $\cdot$ & 4 & \\
\hline Euphrasia pectinata & . & . & . & 5 & . & . & 4 & . & 7 & 20 & 4 & \\
\hline
\end{tabular}

D. s. for alliance Artemisio hololeucae-Hyssopion cretacei

$\begin{array}{llllllllllllll}\text { Scrophularia cretacea } & 33 & 58 & 65 & 45 & 21 & 11 & 17 & 7 & 13 & 16 & \text {. }\end{array}$

$\begin{array}{lllllllllllll}\text { Hyssopus cretaceus } & 80 & 75 & 90 & 95 & 7 & & 4 & 7 & 27 & & & 4\end{array}$

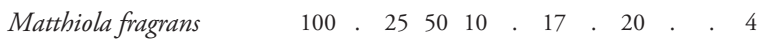

Linaria cretacea

$20.1011 \quad 14$. . . . . .

D. s. for alliance Euphorbio cretophilae-Thymion cretacei

$\begin{array}{lllllllllllll}\text { Artemisia salsoloides } & 20 & \text {. } & 85 & 36 & 34 & 100 & 79 & 7 & & 8 & 8 & 17\end{array}$.

Jurinea stoechadifolia

Euphorbia petrophila

Brassica elongata

. . 18658 . $12 \quad . \quad 12$.

$\begin{array}{ccccccccccc}\cdot & . & 30 & 5 & 93 & 100 & 8 & . & . & 8 & \end{array}$.

D. s. for alliance Centaureo carbonatae-Koelerion talievii

Stipa capillata

Campanula sibirica

Polygala sibirica

Carex humilis

Stipa pennata

Onosma simplicissima

Koeleria talievii

Psephellus marschallianus

Teucrium polium

$\begin{array}{llllllllllll}7 & 5 & 5 & 7 & 3 & . & 25 & 79 & 27 & 72 & 46 & 70\end{array}$ . $\begin{array}{lllllllllll}5 & 5 & 7 & 59 & 42 & 42 & 71 & 20 & 60 & 88 & 61\end{array}$ . . $9 \begin{array}{lllllllll} & 9 & 3 & 17 & 36 & 47 & 24 & 58 & 61\end{array}$ $\begin{array}{lllllllllll}\text {. } & . & 4 & 14 & 17 & 79 & 27 & 60 & 67 & 87\end{array}$ $\begin{array}{lllllllllll}. & 5 & 5 & & & 29 & 86 & & 12 & 25 & 78\end{array}$ $\begin{array}{llllllllllll}7 & . & 23 & 59 & 53 & 58 & 86 & 27 & 64 & 46 & 67\end{array}$ $\begin{array}{lllllllllll}7 & . & 9 & 14 & . & 29 & 50 & 13 & 52 & 13 & 61\end{array}$ $20-366610096100477696$.

\section{D. s. for order Thymo cretacei-Hyssopetalia cretace}

Pimpinella tragium

Gypsophila oligosperma

Cephalaria uralensis

Thymus calcareus

Asperula tephrocarpa

Reseda lutea

Silene supina

Linum pallasianum

Polygala cretacea

Astragalus albicaulis

Genista tinctoria $\begin{array}{llllllllllll}73 & 92 & 90 & 91 & 83 & 95 & 83 & 57 & 93 & 56 & 50 & 37\end{array}$ $\begin{array}{llllllllllll}60 & 50 & 70 & 62 & 76 & 89 & 83 & 93 & 67 & 84 & 79 & 85\end{array}$ $\begin{array}{llllllllllll}27 & 8 & 85 & 74 & 45 & 95 & 79 & 57 & 7 & 8 & 25\end{array}$. $\begin{array}{llllllllllll}60 & . & 5 & 87 & 97 & 100 & 92 & 86 & 93 & 68 & 33 & 96\end{array}$ $\begin{array}{llllllllllll}73 & . & 5 & 90 & 93 & 5 & 88 & 14 & 60 & 52 & 50 & 2\end{array}$ $\begin{array}{llllllllllll}7 & 33 & 5 & 28 & 52 & 74 & 58 & 71 & 13 & 20 & 58 & 9\end{array}$ $33 . \quad \begin{array}{llllllllll}50 & 21 & 5 & 33 & 21 & 40 & 52 & 33 & 4\end{array}$ $27 . \quad . \quad \begin{array}{lllllllll}56 & 62 & 5 & 63 & 79 & 60 & 52 & 54 & 41\end{array}$ $\begin{array}{llllllllllll}20 & 33 & . & 36 & 14 & . & 63 & 57 & 13 & 36 & 25 & 26\end{array}$ $\begin{array}{llllllllllll}40 & 8 & 55 & 21 & 7 & . & 42 & 36 & 13 & 40 & 21 & 54\end{array}$

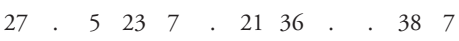

\begin{tabular}{lcccccccccccc}
\hline Consecutive number & 1 & 2 & 3 & 4 & 5 & 6 & 7 & 8 & 9 & 10 & 11 & 12 \\
& 15 & 12 & 20 & 107 & 29 & 19 & 24 & 14 & 15 & 26 & 15 & 46 \\
\hline Taraxacum serotinum & 20 & 17 & 5 & 6 &. &. & 13 & 29 &. & 4 & 8 &. \\
Silene cretacea & 20 &. &. & 5 & 3 &. &. & 14 &. &. &. &. \\
Orthantha lutea & 33 & 25 &. & 20 & 21 &. &. & 21 &. & 40 & 4 &. \\
Hedysarum ucrainicum & 7 &. &. & 7 &. &. &. &. &. &. &. &. \\
Scutellaria supina &. &. &. & 2 & 3 & 21 & 8 &. &. &. & 4 &. \\
Clausia aprica &. &. &. &. &. &. &. &. &. &. &. & 7 \\
Syrenia cana &. &. &. & 2 &. &. &. &. &. &. &. &. \\
Linaria genistifolia &. &. &. & 2 & 3 &. &. &. &. & 4 & 4 &. \\
Schivereckia podolica &. &. &. &. & 3 &. &. &. &. &. &. &.
\end{tabular}

D. s. for class Festuco-Brometea

Festuca valesiaca

Euphorbia seguieriana

Galium ruthenicum

Hieracium virosum

Euphorbia nicaeensis

Stachys recta

Medicago falcata

Agropyron cristatum

Securigera varia

Erysimum diffusum

Stipa pulcherrima

Festuca stricta

Elytrigia stipifolia

Stipa lessingiana

Ajuga chamaepitys

Filipendula vulgaris

Elytrigia intermedia

Hieracium umbellatum

Viola ambigua

Pilosella echioides

Astragalus onobrychis

Potentilla humifusa

Rhaponticoides ruthenica

Onobrychis arenaria

Plantago media

Plantago lanceolata

Achillea setacea

Eryngium campestre

Cota tinctoria

Achillea millefolium

Astragalus subuliformis

Potentilla incana

Potentilla recta

Linum austriacum

Centaurea stoebe

Cleistogenes serotina

Hypericum elegans

Potentilla patula

Stipa zalesskii

. $573 \quad 254320327576$

$\begin{array}{llllllllllll}53 & 8 & 10 & 35 & 14 & 5 & 79 & 79 & 67 & 64 & 75 & 80\end{array}$

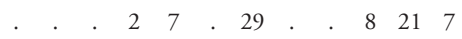

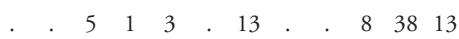

$\begin{array}{llllllllllll}13 & 8 & 10 & 4 & 7 & . & 21 & . & 13 & 16 & 46\end{array}$.

. $\quad 3 \quad 3 \quad 2936 \quad 7 \quad 325422$

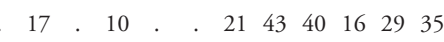

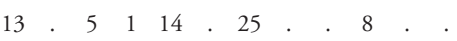

$\begin{array}{llllllllll}25 & 6 & 7 & 29 & 50 & 33 & 12 & 42 & 13\end{array}$

$\begin{array}{llllllllllll}7 & . & 5 & 7 & 10 & 21 & 38 & 43 & 13 & 36 & 17 & 35\end{array}$

. . . . $\quad 38 \quad 7 \quad 7 \quad 4 \quad 21 \quad 2$

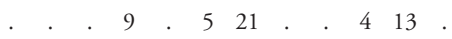

$7 . \quad 3.5 . \quad . \quad$.

$\begin{array}{rrrrrrrrr}. & . & 8 & 3 & 21 & 38 & 14 & 20 & 13\end{array}$.

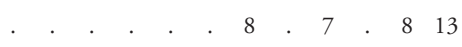

. . . . 4 . . 47 . 17

. . . . $\quad 17 \quad \begin{array}{lllll} & 7 & 4 & 13 & 22\end{array}$

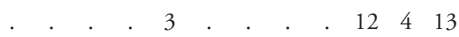

. . . $\quad \begin{array}{llllllll} & 10 & 4 & 4 & & 4 & 17 & 9\end{array}$

. . . . . . 744411

$7 . \quad 3 \quad 3 \quad . \quad \begin{array}{llllll}4 & 29 & 13 & & 17 & 4\end{array}$

. . . . . $\quad 4 \quad 4 \quad 7 \quad 4 \quad 2528$

. . . . $\quad 8 \quad . \quad . \quad 8 \quad 813$

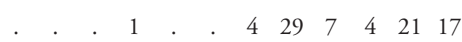

. . . . . $\quad . \quad 4 \quad . \quad . \quad 179$

. $\quad 23$. 2.48 . 48 .

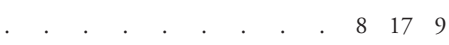

. . $\quad 3 \quad 13.74 \quad 4$.

$\begin{array}{lllllllllll}8 & 5 & 1 & 3 & 11 & 8 & . & 7 & . & 4\end{array}$.

$. \quad . \quad . \quad 13 \quad . \quad 7 \quad 4 \quad 4 \quad 4$.

$7 . \quad 8 . \quad 13 . \quad 124$.

. . . . $4.4 \quad 7 \quad 4 \quad 1311$

. . $\quad 14$. $\quad 7888$.

. . 2 . $25 . \quad 17$. 17

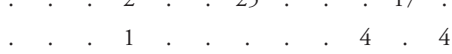

. . $\quad 3.4$. 4217

$\begin{array}{cccccccc}. & . & 7 & 8 & 21 & . & 4 & 17\end{array}$

Other species

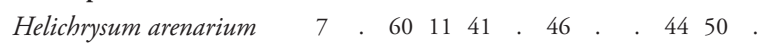

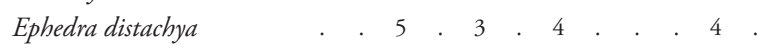

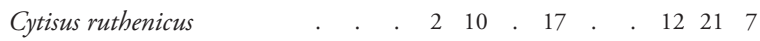




\begin{tabular}{|c|c|c|c|c|c|c|c|c|c|c|c|c|}
\hline Consecutive number & $\begin{array}{c}1 \\
15\end{array}$ & $\begin{array}{c}2 \\
12\end{array}$ & $\begin{array}{c}3 \\
20\end{array}$ & $\begin{array}{c}4 \\
107\end{array}$ & $\begin{array}{c}5 \\
29\end{array}$ & $\begin{array}{c}6 \\
19\end{array}$ & $\begin{array}{c}7 \\
24\end{array}$ & $\begin{array}{c}8 \\
14\end{array}$ & $\begin{array}{c}9 \\
15\end{array}$ & $\begin{array}{l}10 \\
26\end{array}$ & $\begin{array}{l}11 \\
15\end{array}$ & $\begin{array}{l}12 \\
46\end{array}$ \\
\hline Melampyrum arvense & . & 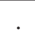 & . & 8 & 3 & . & 13 & 29 & . & 4 & 8 & \\
\hline Centaurea diffusa & - & 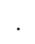 & . & $\cdot$ & 3 & . & 4 & 21 & . & . & 13 & \\
\hline Atraphaxis frutescens & . & . & . & 5 & . & . & . & . & . & . & $\cdot$ & \\
\hline Elytrigia repens & 7 & 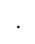 & . & . & . & . & . & . & . & 4 & 13 & \\
\hline Verbascum lychnitis & . & . & . & 2 & . & . & . & . & . & 8 & 4 & 9 \\
\hline Artemisia campestris & . & . & . & . & . & . & . & . & . & . & 8 & 9 \\
\hline Seseli libanotis & . & . & 10 & 2 & . & . & . & . & . & 4 & . & . \\
\hline Melica transsilvanica & . & . & 20 & . & . & . & 4 & . & . & . & 4 & . \\
\hline Poa angustifolia & $\cdot$ & . & 20 & 1 & . & . & 4 & . & . & 16 & 13 & 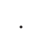 \\
\hline $\begin{array}{l}\text { Krascheninnikovia cera- } \\
\text { toides }\end{array}$ & . & . & 20 & . & . & . & . & . & . & . & . & . \\
\hline Hypericum perforatum & 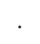 & 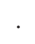 & $\cdot$ & $\cdot$ & . & $\cdot$ & . & . & . & 20 & 13 & $\cdot$ \\
\hline Cichorium intybus & . & 17 & . & . & . & . & . & . & 7 & 4 & 4 & ${ }^{\circ}$ \\
\hline Lotus corniculatus & . & $\cdot$ & . & . & . & . & . & . & . & 4 & . & . \\
\hline Gypsophila fastigiata & ${ }^{\circ}$ & 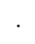 & . & 4 & . & . & . & . & 20 & 8 & $\cdot$ & $\cdot$ \\
\hline Marrubium peregrinum & . & . & . & . & . & . & 4 & . & . & 12 & 13 & . \\
\hline Poa bulbosa & . & $\cdot$ & 5 & 1 & . & . & . & . & . & 20 & 4 & 4 \\
\hline Odontites vulgaris & . & . & . & 4 & . & . & 13 & . & . & . & . & 13 \\
\hline Cotinus coggygria & . & . & . & . & 3 & . & . & . & . & 4 & . & . \\
\hline Calamagrostis epigeios & . & . & . & . & . & . & 8 & . & . & 12 & 8 & . \\
\hline Gypsophila paniculata & $\cdot$ & 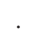 & . & 2 & . & . & 4 & . & . & . & 4 & $\cdot$ \\
\hline Artemisia austriaca & . & . & 15 & 1 & . & . & . & . & . & 12 & 8 & 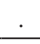 \\
\hline
\end{tabular}

Associations: 1. Artemisio nutantis-Plantaginetum salsae; 2. Erysimo cretacei-Festucetum cretacei; 3. Lepidiomeyeri-Scrophularietumcretacei; 4. Artemisio hololeucae-Polygaletum cretaceae; 5. Euphorbio cretophilae-Jurinetum brachycephalae; 6. Genisto scythicae-Artemisietum salsoloides; 7. Pimpinello titanophillae-Artemisietum salsoloides; 8 . Hedysaro grandiflori-Centauretum carbonatae; 9. Androsacio koso-poljanskii-Caricetum humilis; 10. Bupleuro falcatae-Stipetum capillatae; 11. Gypsophilo oligospermae-Campanuletum sibiricae; 12. Carici humilis-Thymetum calcarei

Classification scheme of chalk outcrop vegetation communities.

Ord. Thymo cretacei-Hyssopetalia cretacei

All. Artemisio hololeucae-Hyssopion cretacei

1 Ass. Artemisio nutantis-Plantaginetum salsae Didukh 1989

2 Ass. Erysimo cretacei-Festucetum cretacei Demina 2011

3 Ass. Lepidio meyeri-Scrophularietum cretacei Demina 2012 (syn. Hedysaro cretacei-Melicetum transsilvanicae Demina 2011)

4 Ass. Artemisio hololeucae-Polygaletum cretaceae Didukh 1989 (syn. Thymo cretacei-Hyssopetum cretacei Didukh 1989, Onosmo tanaiticae-Androsacietum kozo-poljanskii Didukh et al. 1996, Matthiolo fragransi-Atraphaxietum frutescens Demina 2011, Polygalo sibiricae-Hyssopetum cretacei Poluyanov 2012)
All. Euphorbio cretophilae-Thymion cretacei Didukh 1989

5 Ass. Euphorbio cretophilae-Jurinetum brachycephalae Didukh 1989 (syn. Jurineo brachycephalaeHelianthemetum cretophilae Romashchenko et al. 1996; Jurineo brachycephalae-Koelerietum talievii Romashchenko et al. 1996, Scrophulario cretaceiHelianthemetum cretacei Didukh et al. 1996)

6 Ass. Genisto scythicae-Artemisietum salsoloides Sereda 2009

All. Centaureo carbonatae-Koelerion talievii (syn. Carici humilis-Thymion calcarei Averinova 2014)

7 Ass. Pimpinello titanophillae-Artemisietum salsoloides Didukh 1989

8 Ass. Hedysaro grandiflori-Psephelletum carbonatae Averinova 2014 corr. Didukh et al. nom. corr. hoc loco (original name: Hedysaro grandiflori-Centauretum sumensis Averinova 2014: 37-47)

9 Ass. Androsacio koso-poljanskii-Caricetum humilis Korotchenko \& Didukh 1998

10 Ass. Bupleuro falcatae-Stipetum capillatae

11 Ass. Gypsophilo oligospermae-Campanuletum sibiricae Romashchenko et al. 1996 (syn. Scrophulario cretacei-Helianthemetum cretacei Romashchenko et al. 1996)

12 Ass. Carici humilis-Thymetum calcarei Poluyanov 2009

The alliance Artemisio hololeucae-Hyssopion cretacei.

Typical tomillares of this alliance are represented by communities dominated be chamaephyte subshrubs (Thymus calcareus, Hyssopus cretaceus, Pimpinella tragium and Artemisia hololeuca). The communities occupy steep $\left(15-45^{\circ}\right)$ slopes up to $30-50 \mathrm{~m}$, with mostly southern and sometimes eastern exposure. Substrate is a loose, moving chalk. They are confined to the right river banks and are absent in the upland watershed areas. Characteristic features are the processes of denudation and superficial erosion resulting in feeble soil formation or its absence. The plant cover of these communities is quite patchy (30\%). Diagnostic species of these communities are, besides the dominant, Asperula tephrocarpa Popov \& Chrshan., Linum pallasianum Schult, Matthiola fragrans and Scrophularia cretacea.

\section{Artemisio nutantis-Plantaginetum salsae}

Diagnostic species: Plantago maritima Printz, Artemisia nutans Willd., Bassia prostrata (L.) Beck, Convolvulus lineatus $\mathrm{L}$.

Dominant species: Plantago maritima, Artemisia nutans, rarely Artemisia hololeuca, Hyssopus cretaceus.

Among the described associations, this one occupies the most saline substrates. Communities are encoun- 
tered at the foot of the chalk slopes and at their bottom $\left(10-20^{\circ}\right)$ on deluvial, sufficiently coherent sediments, characterised by carbonate-sulfate soil salinisation. Indicators of the salinity are Artemisia nutans, Plantago maritima, Convolvulus lineatus, rarer Bassia prostrata. These are also diagnostic species of this association, which is prevalent in the eastern part of the Luhansk region, on the banks of Siversky Donets' left feeders (Aydar, Derkul, Kamyshna).

\section{Erysimo cretacei-Festucetum cretacei}

Diagnostic species: Erysimum ucrainicum Desf., Festuca cretacea T.I. Popov \& Proskor.

Dominant species: Festuca cretacea, Hyssopus cretaceus, Pimpinella tragium.

The community is abundant in the Rostov region. The association is characterised by a limited species composition. Apart from the dominant species mentioned above, obligate erodiophiles (species associated with soil erosion) with powerful caudex are present (Scrophularia cretacea, Gypsophila oligosperma Krasnova) as well as facultative species with a wide range (Melilotus officinalis (L.) Lam., Poa compressa L., Reseda lutea L., Securigera varia (L.) Lassen) (Demina 2012).

\section{Lepidio meyeri-Scrophularietum cretacei}

Diagnostic species: Hedysarum cretaceum DC., Lepidium meyerii Walp., Hedysarum cretaceum, Artemisia salsoloides.

Dominant species: Artemisia salsoloides, Hedysarum cretaceum, rarely Hyssopus cretaceus, Gypsophila oligosperma, Thymus calcareus, Scrophularia cretacea.

These communities are present on chalk outcrops on the right bank of the Middle Don valley. The association includes two subassociations. Lepidio meyeri-Scrophularietum cretacei typicum is formed at the bottom of the slopes on dense delluvial with signs of carbonate-sulfate salinity. Presence of Lepidium meyerii and sometimes Krascheninnikovia ceratoides is characteristic. Only four species (Hyssopus cretaceus, Artemisia salsoloides, Lepidium meyeri, Pimpinella tragium) have significant roles in coenosis formation (Demina 2012, 2016).

The subassociation L. m.-S. c. hedysaretosum cretacei is restricted to the upper part of slopes with northern aspect. It is formed on chalk washed-off regosols. These are the most "humid" communities, which are confined to northern slopes. Erodiophiles like Melica transsilvanica, Poa compressa and Thesium arvense have a significant share, whereas typical xerophytes are absent. Hedysarum cretaceum and Artemisia salsoloides are most frquent in these communities (Demina 2012, 2016).

\section{Artemisio hololeucae-Polygaletum cretaceae}

Diagnostic species: Artemisia hololeuca, Hyssopus cretaceus, Matthiola fragrans.

Dominant species: Artemisia hololeuca, Artemisia salsoloides, Festuca cretacea, Pimpinella tragium, Thymus calcareus.

These are the most common communities of the alliance. They are confined to Cretaceous eroded steep slopes $\left(15-45^{\circ}\right)$ on the right banks of the Siversky Donets basin with different aspects. These communities are sometimes covered by lime rock of the Upper Eocene in the valleys of the Nagolnaya and Kamyshnaya rivers (Demina 2012). The communities of the association have a low plant cover $(50 \%)$ and a diverse species composition with the same dominant species as mentioned before. For this reason we have selected five associations with rather poor diagnostic blocks. We consider them variants or facies with specific patterns of distribution, primarily with respect to exposure and steepness of slopes and to structure, mobility and density of the substrate. For example, Hyssopus cretaceus and Pimpinella tragium are dominant mostly on moving loose substrate on the northern slopes, while the southern slopes with compressed and strong substrate are covered by Artemisia spp. Under such conditions Thymus calcareus also dominates. It is a characteristic species of the associations of the next alliance. An ongoing involvement of Asperula tephrocarpa, Linum pallasianum and Polygala cretacea Kotov is also characteristic.

The alliance Euphorbio cretophilae-Thymion cretacei includes communities formed on looser substrate with evident organic content both on slight (20\%) and on steeper slopes. The common name "jurineiniky" used for the communities of this alliance indicates the occurrence of Jurinea spp. (J. stoechadifolia s.l., J. arachnoidea Bunge), which are diagnostic species of this association. Euphorbia petrophila, Helianthemum canum (L.) Hornem. and Thymus calcareus subshrubs also contribute to the communities of this alliance. Artemisia salsoloides is dominant on the steeper rocky slopes. With approximately $40-60 \%$, the plant cover of these communities is higher than in the previous alliance.

\section{Euphorbio cretophilae-Jurinetum brachycephalae}

Diagnostic species: Euphorbia petrophila, Helianthemum canum, Jurinea stoechadifolia.

Dominant species: Helianthemum canum, Jurinea stoechadifolia, Thymus calcareus.

This association is common in the South of the research territory, on the right banks of the Siversky Donets River and its left tributaries. The communities occur on steep and moderately steep slopes (up to $15^{\circ}$ ) in areas adjacent to upland and hilltops. Its communities are characterised by the dominance of the orthotropic chasmophyte $J u$ - 
rinea stoechadifolia, among which the plagiotropic Helianthemum canum and Thymus calcareus occur. The plant cover can reach $70 \%$.

\section{Genisto scythicae-Artemisietum salsoloides}

Diagnostic species: Odontarrhena tortuosa (Waldst. \& Kit. ex Willd.) C. A. Mey., Artemisia salsoloides, Rhaponticoides taliewii, Diplotaxis cretacea Kotov, Euphorbia petrophila, Genista scythica Pacz., Jurinea stoechadifolia, Leontodon biscutellifolius DC., Linaria vulgaris Mill., Linum hirsutum, Linum tenuifolium L., Reseda lutea.

Dominant species: Thymus calcareus, Genista scythica, Jurinea stoechadifolia, Artemisia salsoloides.

Communities are gappy (plant cover 40-60\%) and confined to the middle and upper parts of river valleys slopes (with $30-45^{\circ}$ steepness), typically with southern, southeastern or southwestern exposure.

It is a quite rare association with specific diagnostic species. Outcrops along the Tuzlov River in the Rostov region are the only location of petrophytic communities with occurrences of Genista scythica in Russia (Sereda 2008). In Ukraine Genista scythica grows only on the Donetsk Ridge and does not occur within the research territory.

The communities of the alliance Centaureo carbonataeKoelerion talievii are confined to places with chalk deposits and outcrops. Unlike in case of the alliance mentioned above, carbonaceous rocks are immobile and very dense, but the soil is not formed (regosols) or undifferentiated (rendzinas). The plant cover is much denser, ranging from 30 to $70 \%$. Dominant species are Thymus calcareus, Carex humilis, C. pediformis, Onosma simplicissima, Androsace villosa (Ovcz.) Fed. and Hedysarum grandiflorum. Typical for cretaceous communities are the following species: Linum pallasianum L., L. hirsutum, Teucrium polium L., Brassica elongata Ehrh., Thymus calcareus, Onosma simplicissima, Silene supina, Psephellus marschallianus (Spreng.) K. Koch. Steppe species (Carex humilis, Stipa capillata L.) are also part of the floristic composition (Didukh \& Chusova 2014). However, there are no such typical carbonatophiles as Hyssopus cretaceus, Scrophularia cretacea and Matthiola fragrans, and occurrence of Artemisia hololeuca and $A$. salsoloides is limited.

This peculiarity indicates that it may be included in the order Stipo pulcherrimae-Festucetalia pallentis, but to prove this argument, it is necessary to analyse larger samples from other regions. Given that similar groups dominated by Jurinea stoechadifolia, Pimpinella tragium ssp. titanophylla and Thymus dimorphus Klokov \& Des.-Shost. are distributed more widely, particularly on limestone in the steppe zone of Ukraine and Moldova, the final solution for this issue would be possible through broader analysis. As for now, the presence and dominance of hyper-car- bonatophiles and carbonatophiles (Helianthemum canum, Thymus calcareus, Brassica elongata, Euphorbia petrophila, Koeleria talievii, Hedysarum grandiflorum, Asperula tephrocarpa) and the minor role of grass and typical steppe species of the order Festucetalia valesiacae (Medicago falcata L., Agrimonia eupatoria L., Veronica incana L., Ranunculus polyanthemos L., Achillea millefolium L., Potentilla argentea L., Falcaria vulgaris Bernh., Plantago media L., Elytrigia intermedia (Host) Nevski, Carex praecox Schreb., Tragopogon dubius (Jacq.) Vollm.) suggest to subsume these communities in the order Thymo cretacei-Hyssopetalia cretacei.

An important feature of this alliance is the quite strong floristic variability of its communities. They are based on the diagnostic species of the alliance, but in some associations, the diagnostic species are not clearly marked. That is why it is quite difficult to distinguish such communities solely by the floristic composition.

\section{Pimpinello titanophillae-Artemisietum salsoloides}

Diagnostic species: Artemisia salsoloides, Jurinea arachnoidea, Linum austriacum L., Polygala cretacea.

Dominant species: Artemisia salsoloides, Hedysarum cretaceum, Onosma simplicissima L., Thymus calcareus.

The communities are visually differentiated from a background of gray aspect due to the dominance of tall $(60 \mathrm{~cm})$, dense dark green stands of $A$. salsoloides. They are sporadically abundant in the middle of gentle $\left(20^{\circ}\right)$ slopes along the banks of left tributaries (Krasna, Aidar, Derkul, Kamyshna) of the Siversky Donets.

\section{Hedysaro grandiflori-Psephelletum carbonatae}

Diagnostic species: Adonis vernalis L., Allium inaequale Janka, Asperula cynanchica L., Carex humilis, Hedysarum grandiflorum, Phlomis herba-venti (Willd.) Maire ex De Filipps, Pimpinella saxifraga L., Salvia verticillata L., Stipa capillata, Stipa pennata L., Thymelea passerina (L.) Coss. \& Germ.

Dominant species: Carex humilis, Festuca valesiaca Gaudin, Gypsophila oligosperma, Thymus calcareus.

As Averinova (2014) notes, communities are distributed on chalk outcrops of relatively gentle $\left(2-10^{\circ}\right)$ slopes of ravines and river valleys with mainly southern exposure. The substrate is a chalky fine earth with broken rock. The association may be diagnosed by the presence of a number of species. Its communities are described from the Belgorod region (Russian Federation).

The choice of Psephellus sumensis (Kalen.) Greuter. as diagnostic species is false because the race distinguished by M. Klokov is psamophytic and bound to boreal sand and pine forest communities. It is replaced by Psephellus carbonatus (Klokov) Greuteron on calcareous soil. Both species are included in the complex P. marschallianus s.l. 


\section{Androsacio koso-poljanskii-Caricetum humilis}

Diagnostic species: Androsace villosa.

Dominant species: Androsace villosa, Artemisia hololeuca, Carex humilis, Thymus calcareus.

The association is confined to rather steep $\left(30-50^{\circ}\right)$ slopes with different exposures. Its communities occur on a feebly (up to several centimetres) developed rendzina high in carbonate. They are characterised by a high species richness and the presence of species with well-developed sod (Carex humilis, Festuca valesiaca).

\section{Bupleuro falcatae-Stipetum capillatae}

Diagnostic species: Carex humilis, Koeleria talievii Lavren-

ko, Poa bulbosa L., Vincetoxicum hirundinaria Medik.

Dominant species: Carex humilis, Stipa capillata, Thymus calcareus.

Communities occur at the bottom of gentle slopes and in depressions of slopes with moderate soil moisture. The association is contiguous to communities of the order Festucetalia valesiacae. Its communities are diagnosed by the dominance of Stipa capillata with species of the order Thymo cretacei-Hyssopetalia cretacei being present. The plant cover is more than $60 \%$.

\section{Gypsophilo oligospermae-Campanuletum sibiricae}

Diagnostic species: Bromopsis riparia (Rehmann) Holub, Campanula sibirica L., Carex pediformis, Galatella villosa (L.) Rchb. f., Hieracium virosum Pall., Inula aspera Poir., Koeleria pyramidata (Lam.) P. Beauv., Nonea pulla DC., Salvia nutans L., Veronica incana, Veronica spicata L.

Dominant species: Astragalus albicaulis DC., Bromopsis riparia, Carex humilis, Psephellus marschallianus, Galatella villosa, Festuca valesiaca, Gypsophila oligosperma, Helianthemum canum, Onosma simplicissima, Salvia nutans, Stipa capillata, Stipa pennata, Thymus calcareus. The communities develop on slightly eroded chalk slopes and hilltops of the chalk mountains, on steppified sites with Cretaceous basis. The association is quite common and widespread. In general the plant cover may reach $80 \%$ due to the presence of Carex humilis and representatives of the Poaceae family. The well-developed vegetation cover prevents surface erosion. The communities are observed to have a high species diversity. Stipa spp. or other prevalent grasses act as dominant species. However, due to the general xerophytism and significant contribution of carbonatophiles, these communities belong to the order Thymo cretacei-Hyssopetalia cretacei. The association most commonly occupies areas transitional to the steppe vegetation adjacent to upland or steep $\left(15-30^{\circ}\right)$ southern slopes. In such places the plant cover contributes to soil accumulation. This association also includes communities with dominance of Carex pediformis, which is an arctic-boreal species. It is on the southern edge of its area here and is found only in a few exclaves. The coenotic range of the communities is quite narrow (Golitsyin 1958, Didukh \& Chusova 2014).

\section{Carici humilis-Thymetum calcarei}

Diagnostic species: Anthericum ramosum L., Bupleurum falcatum, Carex humilis, Euphrasia pectinata Ten., Helianthemum nummularium (L.) Mill., Pilosella officinarum Vaill., Vincetoxicum hirundinaria, Viola rupestris F.W. Schmidt

Dominant species: Androsace villosa, Thymus calcareus.

This association combines communities that are typical for the Upper Oskol Region on slightly sodded chalky slopes with dominance of calciphilous shrubs and subshrubs. Its communities often occupy the most elevated relief elements, which are well warmed up, i.e. the upper and middle parts of steep (up to $40^{\circ}$ ) slopes with exposure to the South. They are less common on northern slopes and gentle hilltops (Poluyanov 2012).

To estimate separated syntaxa in the general classification system of xerophilous grass-undershrub vegetation, we have processed the entire array of relevés and built a tree diagram on the basis of species composition similarity. As a result, there are six allocated clusters (Figure 3), two of which (1 and 2) correspond to alliances of the order Thymo cretacei-Hyssopetalia cretacei. Relevés of steppe vegetation (order Festucetalia valesiacae) matched the clusters 3-5 and relevés of sandy steppes (class Koelerio-

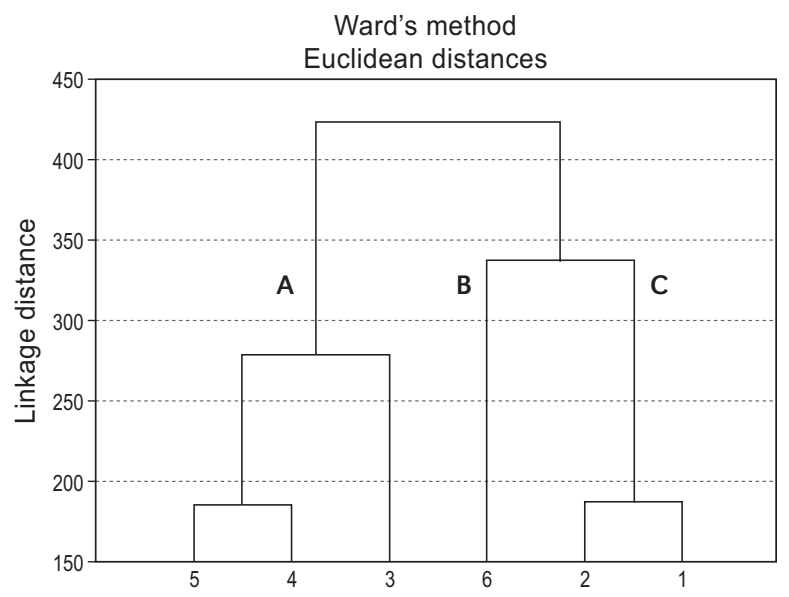

Figure 3: Distribution of Cretaceous outcrop vegetation in relation to the steppe vegetation. A - Ord. Festucetalia valesiacae; B - Cl. KoelerioCorynephoretea (all. Festucion vaginatae); $\mathrm{C}$ - Ord. Thymo cretaceiHyssopetalia cretacei.

Slika 3: Razporeditev vegetacije krednih izdankov glede na stepsko vegetacijo. A - red Festucetalia valesiacae; B - razred KoelerioCorynephoretea (all. Festucion vaginatae); C - red Thymo cretacei-Hyssopetalia cretacei. 
Corynephoretea) cluster 6 . Thus, this analysis shows that the communities of the order Thymo cretacei-Hyssopetalia cretacei differ from the Festucetalia valesiacae communities as a Koelerio-Corynephoretea in their floristic composition. All these communities have in common the predominance of xerophilous species; however, grasses (Festuca, Stipa) dominate in Festucetalia valesiacae and Koelerio-Corynephoretea communities, whereas chasmophilous plants are dominant in the order Thymo cretacei-Hyssopetalia cretacei.

The position of Cretaceous outcrop vegetation communities within the modern system of syntaxonomical classification is quite debatable and depends on the interpretation of the size of different syntaxa. Considering Festuco-Brometea in a fairly wide range, it should be noted that calciphyte communities are common in the steppe zone and have been in direct contact with zonal vegetation so that some species of the class Festuco-Brometea are present in those coenoses. But in general, these events are quite random with low class constancy (I-II). Inclusion of the order Thymo cretacei-Hyssopetalia cretacei into the class Festuco-Brometea can be considered as substitution of Stipo pulcherrimae-Festucetalia pallentis Pop 1968 in the East. However, the difference in ecological conditions between them is that the first is common on dense carbonates (gypsum, dolomite, limestone) and the latter - on idiomorphic soils with different densities - often on loose and mobile regosols. In this respect, the Thymo cretacei-Hyssopetalia cretacei communities may be regarded as similar to Koelerio-Corynephoretea Klika in Klika et Novák 1941, and Stipo pulcherrimae-Festucetalia pallentis as an analogue of Sedo-Scleranthetea $\mathrm{Br}$.-Bl 1955. They are similar in structure and growing conditions to the Mediterranean communities of the class Drypidetea spinosae Quezel 1964, where Mucina et al. (2016) included Crimean-Caucasian syntaxa of Onosmo polyphyllae-Ptilostemetalia Korzhenevsky 1990. But the location and status of this order has not been fully established yet. All of the above shows that a number of syntaxonomical issues are still unresolved and require broader discussion.

\section{Acknowledgement}

The autors are very grateful to Dr. Orsolya Valkó for editing and handling the manuscript, to Dr. Aiko Huckauf and Andriy Mosyakin for English proofreading and linguistic edition. We also thank two anonymous reviewers for the valuable comments and recommendations.

\section{Conclusions}

We provided a comprehensive syntaxonomic survey of Cretaceous outcrop vegetation from south-western foothills of the Central Russian Upland. Based on relevés from Ukraine and the Russian Federation, we could conclude that all communities belong to the order Thymo cretacei-Hyssopetalia cretacei and are clearly separated from other steppe communities. The order Thymo cretacei-Hyssopetalia cretacei includes twelve associations belonging to the three alliances Artemisio hololeucae-Hyssopion cretacei, Euphorbio cretophilae-Thymion cretacei and Centaureo carbonatae-Koelerion talievii.

\section{References}

Averinova, E. 2005: Calcifitic steppe communities of the Seim River basin (within the Kursk region). Vegetation of Russia 7: 39-49. [in Russian]

Averinova, E. 2011: Calcareous vegetation of the Natural park "Rovenskyi" (Belgorodregion). Bulletin of the Bryansk State University 4: 60-65. [in Russian]

Averinova, E. 2012: Calcareous vegetation of the Natural reserve "Divnogorje" (Voronezh region). Bulletin of the Bryansk State University 4 (2): 87-89. [in Russian]

Averinova, E. 2014: Communities with Hedysarum grandiflorum Pall. within the territory of the Middle Russian upland. Bulletin of the Bryansk Departmentof the Russian Botanical Society 1 (3): 37-47. [in Russian]

Baranov, A. (ed.) 1969: Atlas of the USSR. Main Department of Geodesy and Cartography under the Council of Ministers of the USSR, Moscow, 199 pp. [in Russian]

Bohn,U. \& Neuhäusl, R. 2000: Map of the Natural Vegetation of Europe / Karte der Natürlichen Vegetation Europas: Part 2 / Teil 2: Legend / Legende (Folded Maps). BfN-Schriftenvertrieb, 156 pp.

Chytrý, M., Tichý, L., Holt, J. \& Botta-Dukát, Z. 2002:

Determination of diagnostic species with statistical fidelity measures. Journal of Vegetation Science 13: 79-90.

Cvelev, N. N., Alekseev, Yu. E., Geltman, D. V., Grabovskaya (Borodina), A. E., Grintal, A. R., Gusev, Yu. D., Egorova, T. V., Ikonnikov, S. S., Krupkina, L. I., Linchevskyi, I. A., Medvedeva, N. A., Mordak, E. V., Mosyakin, S. L., Nikitin, V. V., Olyanitskaya, L. G., Pobedimova, E. G., Sennikov, A. I., Skvortsov, A. K., Cmolyaninova, L. A., Vasilchenko, I. T. \& Vasil'ev, I. V. 1996: Flora of the East Europe (Vol. 9). St. Petersburg, 456 pp. [in Russian]

Demina, O. N. 2011: Regularity of distribution and development of steppe plant formation in Don River basin (within the Rostov region), Moscow: 50 pp. [in Russian]

Demina, O. N. 2012: New association of hyssop and thyme steppes at Donetsk Ridge. Proceedings of the Samara Scientific Center of the Russian Academy of Sciences 1(4): 998-1003. [in Russian]

Demina, O. N. 2014: “The Don steppe preserve" and the Don basins' east pontic. Southern Federal University, Rostov-on-Don, 63 pp. 
Demina, O. N. 2016: East pontic steppesand its territorial preserve. "IP Skorokhodov V.A.", Moscow, 64 pp. [in Russian]

Didukh, Ya. P. 1981: Tomiliars of the Mountain Crimea. Ukrainian Botanical Journal 38(4): 18-23. [in Ukrainian]

Didukh,Ya.P. 1989: Floristic classification of communities of "Hyssopus flora”. Ukrainian Botanical Journal 46(6): 16-21. [in Ukrainian]

Didukh, Ya. P. \& Korotchenko, I. A. 1996: The steppe vegetation on the southern part of the Left-Bank Forest-Steppe of the Ukraine. I. Class Festucetea vaginatae and Helianthemo-Thymetea. Ukrainian phytosociologic collection 2: 56-63. [in Ukrainian]

Didukh, Ya. P. \& Korotchenko, I. A. 1997: The steppe vegetation on the southern part of the Left-Bank Forest-Steppe of the Ukraine. II. Class Festuco-Brometea. Ukrainian phytosociologic collection 1 (6): 20-39. [in Ukrainian]

Didukh, Ya. P. (ed.) 2009: Green Data Book of Ukraine. Al'terpres, Kyiv, 448 pp. [in Ukrainian]

Didukh, Ya. P. \& Chusova, O. O. 2014: Rare xerophytic steppe communities and biotopes in the Krasna river valley (Lugansk region). Ukrainian Botanical Journal 71(3): 275-285. [in Ukrainian]

Dobrochayeva, D. N., Kotov, M. I., Prokudin, Yu. N., Barbarych, A. I., Chopik, V. I., Protopopova, V. V., Morozyuk, S. S., Dubovik, O. N., Zyman, S. N., Shelyag-Sosonko, Yu. R., Myakushko, T. Ya., Zaveruha, B. V., Katina, Z. F., Skrypnik, N. P., Kritskaya, L. I., Lypa, A. L., Mrinskiy, O. P., Alekseev, Yu. E., Skvortsov, A. K., Olyaniskaya, L. G., Krasnova, A. H., Tveretinova, V. V., Slyusarenko, L. P., Shymkus, G. T., Didukh, Ya. P., Glagoleva, N. G., Sipaylova, L. M., Ilinskaya, A. F., Chernyavskiy, A. V., Fedoronchuk, N. S., Ivchenko, I. S., Boyko, T. G., Verenko, A. V. \& Ornst, E. Y. 1987: The determinant of higher plants of Ukraine. Naukova dumka, Kyiv, 548 pp. [in Ukrainian]

Euro+Med (2006): Euro+Med PlantBase - the information resource for Euro-Mediterranean plant diversity. Published on the Internet http:// ww2.bgbm.org/EuroPlusMed [last accessed on 2010]

Golitsyin, S. V. 1936: To the botanical-geographical characteristics of the south-west of Kursk region. Proceedings of Voronezh State University 9 (1): 98-144. [in Russian]

Golitsyin, S. V. 1958: Carex pediformis C.A. Mey on the South of Central Russian Upland. Botanical Journal 43 (12): 1740-1748. [in Russian]

Golitsyin, S. V. 1965: "Lowered Alps" and chalk vegetation communities with Hyssopus of the Central Russian Upland. Voronezh University Publishing House, Voronezh, 16 pp. [in Russian]

Golitsyin, S. V. 1970: Relict flora and vegetation. In Golitsyin S. V., Doronin Yu. A.: Monuments of nature of the Voronezh region. Central Black Earth Book Publishing House, Voronezh, pp. 107-119. [in Russian]

Golitsyin, S. V., Doronin, Yu. A. \& Matyunenko, V. V. 1963: Hedysarum cretaceum Fisch. in the western part of its range. News of the Voronezh branch of the Voronezh Botanical Society: 29-34. [in Russian]

Grosset, G. O. 1935: On the age of the plains relic flora of the European part of the USSR. Zemlevedeniye 37 (3): 185-234. [in Russian]

Hennekens, S. M. \& Schaminee, J. H. 2001: TURBOVEG, a comprehensive data base management system for vegetation data. Journal of Vegetation Science 12: 589-591.
Honcharenko, I. 2000: Vegetation chalk outcrops of the Sumy geobotanical district / Fitosotsiolohiya. 100 years of scientific direction, Kyiv, 163-169. [in Ukrainian]

Hryn', F. O. 1938: To the question of the dynamics of chalk outcrops vegetation. Ukrainian phytosociologic collection 2: 89-110. [in Ukrainian]

Hryn', F. O. 1973: Chalk outcrops vegetation. In: Barbarych A. et al. (eds.): Vegetation of the UkrSSR: Steppe, stony outcrop, sand. Naukova dumka, Kyiv, pp. 336-356. [in Ukrainian]

Hrytsenko, A. (ed.). 2004: Environmental Atlas of Luhansk region. LODA, Luhansk: 167 pp. [in Ukrainian]

Karandeeva, M. 1957: Physiography of Europian part of USSR. Moscow state university, Moscow, 314 pp. [in Russian]

Kleopov, Yu. D. 1930: About the Mariupol flora in connection with the relic issues in Ukraine. Bulletin of the Kiev Botanical Garden 1: 20 pp. [in Ukrainian]

Klokov, M. V. \& Dobrochayeva, D. N. 1974: A note about Helianthemum canum and its related species. Systematics news of higher and lower plant-forms. Naukova dumka, Kyiv, pp. 67-77 [in Ukrainian]

Kondratyuk, E. N., Burda, R. I., Chupryna, T. T. \& Khomyakov, M.T.1998: Lugansk State Reserve. Vegetable world. Naukova dumka, Kyiv, 188 pp. [in Russian]

Kozo-Polyanskyi, B. M. 1910: On the study of Voronezh flora. Russian Botanical Journal 4-6: 33-43. [in Russian]

Kozo-Polyanskyi, B. M. 1925: Botanical Impressions from Galichya Mountain. Izvestiya of the Voronezh Local Lore Society 3. [in Russian]

Kozo-Polyanskyi, B. M. 1931: In the land of living fossils. State educational and pedagogical publishing house, Moscow, 184 pp. [in Russian]

Lavrenko, E. 1961: On the development of some coenotic types of the flora of Ancient Middle-earth in connection with Alpine orogenesis. Proceedings of Tashkent State University 187 (38): 17-27. [in Russian]

Lavrenko, E. M. 1932: Über die Entwicklungsyentren der Flora und das Altrer des ukrainischen Endemismus. Die Quartärperiode 4: 27-43.

Litvinov, D. 1891: Geobotanical notes on the flora of European Russia. Moscow, 123 pp. [in Russian]

Litvinov, D. 1902: On the relic character of the stony slopes flora of European Russia. Proceedings of Botanical Museum of Academy of Science 1: 67. [in Russian]

Mucina, L., Bueltmann, H., Dierßen, K., Theurillat, J.-P., Raus, T., Čarni, A., Šumberová, K., Willner, W., Dengler, J., Gavilán, R., Chytrý, M., Hájek, M., Di Pietro, R., Iakushenko, D., Pallas, J., Daniëls, F., Bergmeier, E., Santos Guerra, A., Ermakov, N. \& Tichý, L. 2016: Vegetation of Europe: Hierarchical floristic classification system of vascular plant, bryophyte, lichen, and algal communities. Applied Vegetation Science 19 (Suppl. 1): 3-264.

Pachos'kyy, J. K. 1910: The main features of the development of the flora of south-western Russia. Notes of the Novorossiysk Society of Naturalists (Supplement to the edition 34): 430 pp. [in Russian]

Poluyanov, A. V. 2009: Petrofitic feather-grass and thyme steppes in the South-East of the Kursk Regiion (within the limits of the 
Oskol River basin). Vegetation of Russia. St. Petersburg: 49-62 pp. [in Russian]

Poluyanov, A. 2012: Grass vegetation of the Kursk region (syntaxonomy and protection issues) In: Poluyanov, A. \& Averinova, A. (eds.): Kursk State University, Bryansk State University, 276 pp. [in Russian]

Roleček, J., Tichý L., Zelený, D., Chytrý, M. 2009: Modified TWINSPAN classification in which the hierarchy respects clusterheterogeneity. Journal of Vegetation Science 20: 596-602.

Romashchenko, K., Didukh, Ya. \& Solomaha, V. 1996: Syntaxonomy of Helianthemo-Thymetea cl. nov. of chalk outcrops vegetation of south-estern Ukraine. Ukrainian phytosociologic collection 1: 49-62. [in Ukrainian]

Semenova-Tian-Shanskaya, A. M. 1954: Plant biology and vegetation dynamics of Cretaceous outcrops along the Derkul River. Proceedings of Academy of Sciences of the USSR 9: 578-645. [in Russian]

Sereda, M. M. 2003: Syntaxonomy of petrophytic steppes of the Don river basin. Stavropol, 19 pp. [in Russian]

Sereda, M. M. 2008: New association of petrophyte vegetation of Northern Pryazovie. Vegetation of Russia, 12: 62-67. [in Russian]

Sereda, M. M. 2009: Chalk outcrops vegetation of the Don River in Rostov region. Vegetation of Eastern Europe: Classification, ecology and protection: 201-205.[in Russian]

Shelyag-Sosonko, Yu. R., Dydukh, Ya. P. \& Dubyna, D. V. 1991: Checklist of vegetation of Ukraine. Naukova dumka, Kyiv, 272 pp. [in Russian]

Szafer, W. 1928: Trzeciorzędowe rośliny górskie na wale scytyjskim w ostoi podolsko-wołyńskiej. Acta Societatis Botanicorum Poloniae 1 (2): 97-119.
Taliev, V. 1897: On the issue of relic plants of the glacial period. Proceedings of the Kharkov Society of Naturalists 31: 127-241. [in Russian]

Taliev, V. 1904: Chalk outputs vegetation of Southern Russia. I. Proceedings of the Society of Naturalists at Kharkov University 39(1): 81-238.

Taliev, V. 1905: Chalk outputs vegetation of Southern Russia. II. Proceedings of the Society of Naturalists at Kharkov University 4(1): 1-282.[in Russian]

Tichý, L. 2002: JUICE, software for vegetataion classification. Journal of Vegetation Science 13: 451-453.

Tkachenko,V.S., Didukh,Ya. P., Henov, A. P., Dudka, I. O., Wasser, S. P., Boyko, M. F., Vetrova, Z. I., Navrotskaya, I. L., Partika, L. Ya., Heluta, V. P., Smyk, L. V., Tyhonenko, Yu. Ya., Merezhko, T. O., Burdyukova, L. I. \& Soldatova, I. M. 1998: Ukrainian natural steppe reserve. Vegetable world. Fitosotsiotsentr, Kyiv, 280 pp. [in Ukrainian]

Tsinher, V. 1885: Collection of information on the flora of Central Russia. University Printing House, Moscow, 517 pp. [in Russian]

Tutin, T. G. (ed.) 1968-1993: Flora Europaea (Vol. 1-5.), Cambridge University Press.

Vakarenko, L. P. \& Dubyna, D.V. 2006: Rarity phytocenotic fund of shrub and herbaceous vegetation of southern Ukraine. Chornomorski Botanical Journal 2 (1): 108-115. [in Ukrainian]

Weber, H. E., Moravec, J. \& Theurillat, J.-P. 2000: International Code of Phytosociological Nomenclature. 3rd edition. Journal of Vegetation Science 11: 739-768.

Weather in Ukraine (2014). Published on the Internet. http://ukraine. pogoda360.ru [last accessed on 2017] 
Appendix 1a: Characteristic table of syntaxa of the alliance Artemisio hololeucae-Hyssopion cretacei.

Priloga 1a: Tabela sintaksonov zveze Artemisio hololeucae-Hyssopion cretacei.

\begin{tabular}{lccccccccccccccccccccc}
\hline Consecutive number & 1 & 2 & 3 & 4 & 5 & 6 & 7 & 8 & 9 & 10 & 11 & 12 & 13 & 14 & 15 & 16 & 17 & 18 & 19 & 20 & 21 \\
\hline Total vegetation cover (\%) & 60 & 15 & 40 & 20 & 50 & 45 & 20 & 50 & 40 & 25 & 70 & 70 & 50 & 40 & 20 & 30 & 50 & 60 & 40 & 50 & 50 \\
Slope $\left(^{\circ}\right)$ & 10 & 5 & 5 & & 25 & 30 & 45 & 25 & 5 & & 15 & & 25 & & & & & 30 & 30 & 20 & 45 \\
Aspect $\left({ }^{\circ}\right)$ & 5 & 90 & 180 & 135 & 180 & 135 & 90 & 180 & 180 & 45 & & & & 90 & 90 & 180 & 90 \\
Species richness & 14 & 11 & 15 & 19 & 17 & 15 & 15 & 13 & 11 & 14 & 17 & 9 & 13 & 13 & 11 & 14 & 20 & 16 & 15 & 17 & 16
\end{tabular}

D. s. of ass. Artemisio nutantis-Plantaginetum salsae

Plantago maritima

Artemisia nutans

$\begin{array}{llllllllll}3 & 1 & 4 & 4 & 3 & 1 & 2 & 2 & 1 & 1\end{array}$

Bassia prostrata

Convolvulus lineatus

D. s. of ass. Artemisio hololeucae-Polygaletum cretacei

Artemisia hololeuca

$\begin{array}{llllllllll}4 & 2 & 3 & 2 & 1 & 1 & 1 & 1 & 1 & 1\end{array}$

D. s. of alliance Artemisio hololeucae-Hyssopion cretacei

Scrophularia cretacea

Hyssopus cretaceus

Matthiola fragrans

Linaria cretacea

D. s. of alliance Euphorbio cretophilae-Thymion cretacei

Brassica elongata

Artemisia salsoloides

D. s. of alliance Centaureo carbonatae-Koelerion talievii

Polygala sibirica

Carex humilis

Onosma simplicissima

Koeleria talievii

Teucrium polium

$\begin{array}{ccccccccccccccccccccc}. & . & . & . & . & 1 & 1 & . & . & 1 & . & . & 1 & 2 & 2 & 2 & . & 1 & . & 1 & . \\ . & . & 1 & . & 3 & 3 & 2 & 2 & 3 & 2 & 1 & 3 & 2 & 2 & 2 & 3 & 2 & 1 & 1 & 3 & 1 \\ 1 & 1 & 1 & 1 & 1 & 1 & 1 & 1 & 1 & 1 & 1 & . & . & 1 & 1 & 2 & 1 & 1 & 1 & 1 & . \\ . & . & . & 1 & 1 & . & 1 & . & . & . & . & . & . & . & . & . & . & . & 2 & . & .\end{array}$

D. s. of order Thymo cretacei-Hyssopetalia cretacei

Pimpinella tragium

Gypsophila oligosperma

Cephalaria uralensis

Thymus calcareus

Asperula tephrocarpa

Reseda lutea

Silene supina

Linum pallasianum

Polygala cretacea

Astragalus albicaulis

Genista tinctoria

Taraxacum serotinum

Silene cretacea

Orthantha lutea

Hedysarum ucrainicum

D. s. of class Festuco-Brometea

Euphorbia seguieriana

Euphorbia nicaeensis

Medicago falcata

Astragalus onobrychis

Plantago lanceolata 
Localities: 01 - vil. Stril'tsivka, Milovs'kyy district; Lugansk reg.; Ukraine; 06.1987 (Ya. Didukh)

Localities: 02 - vil. Stril'tsivka, Milovs'kyy district; Lugansk reg.; Ukraine; 27.06.1987 (Ya. Didukh)

Localities: 03 - Bilovodsk, Lugansk reg.; Ukraine; 06.1987 (Ya. Didukh)

Localities: 04 - right bank of Aidar River, vil. Rybiancevo, Lugansk reg.; Ukraine; 06.1987 (Ya. Didukh)

Localities: 05 - vil. Kamyshivka, Lugansk reg.; Ukraine; 06.1987 (Ya. Didukh)

Localities: 06 - Horodysche, Lugansk reg.; Ukraine; 06.1987 (Ya. Didukh)

Localities: 07 - vil. Stril'tsivka, Milovs'kyy district;Lugansk reg.; Ukraine; 06.1987 (Ya. Didukh)

Localities: 08 - Serebryanka, Donetsk reg.; Ukraine; 06.1987 (Ya. Didukh)

Localities: 09 - Bilovodsk, Lugansk reg.; Ukraine; 06.1987 (Ya. Didukh)

Localities: 10 - vil. Stril'tsivka, Milovs'kyy district;Lugansk reg.; Ukraine; 06.1987 (Ya. Didukh)

Localities: 11 - Derkul River, Markivka, Lugansk reg.; Ukraine; 3.07.1987 (Ya. Didukh)

Localities: 12 - vil. Razdollya, Lugansk reg.; Ukraine; 16.07.2009 (L. Borovyk)

Localities: 13 - Nova Duvanka, Lugansk reg.; Ukraine; 06.1987 (Ya. Didukh)

Localities: 14 - vil. Zorykivka, Lugansk reg.; Ukraine; 24.06.2009 (L. Borovyk)

Localities: 15 - Dubovyy ravine, Milovs'kyy district; Lugansk reg.; Ukraine; 22.06.2008 (L. Borovyk)

Localities: 16 - vil. Stril'tsivka, Milovs'kyy district; Lugansk reg.; Ukraine; 23.08.2011 (L. Borovyk)

Localities: 17 - "Striltsivskyi steppe” (Luhansk Natural Reserve); Lugansk reg.; Ukraine; 6.07.2012 (L. Borovyk)

Localities: 18 - Derkul River, Markivka, Lugansk reg.; Ukraine; 3.07.1987 (Ya. Didukh)

Localities: 19 - Krasna River, Svatovo, Lugansk reg.; Ukraine; 06.1987 (Ya. Didukh)

Localities: 20 - Bilovodsk, Lugansk reg.; Ukraine; 06.1987 (Ya. Didukh)

Localities: 21 - Oskil River, Dvorichna, Kharkiv reg.; Ukraine; 06.1995 (Ya. Didukh) 
Appendix 1a (extension): Characteristic table of syntaxa of the alliance Artemisio hololeucae-Hyssopion cretacei.

Priloga 1a (nadaljevanje): Tabela sintaksonov zveze Artemisio hololeucae-Hyssopion cretacei.

\begin{tabular}{lccccccccccccccccccccc}
\hline Relevé number & 1 & 2 & 3 & 4 & 5 & 6 & 7 & 8 & 9 & 10 & 11 & 21 & 12 & 13 & 22 & 14 & 23 & 15 & 18 & 19 & 24 \\
\hline Species richness & 8 & 6 & 4 & 21 & 7 & 16 & 6 & 52 & 31 & 4 & 11 & 14 & 10 & 10 & 15 & 13 & 16 & 11 & 10 & 12 & 15 \\
Slope $\left(^{\circ}\right)$ & 50 & 70 & 70 & & & & & & & & 7 & & 5 & 3 & & 3 & & 5 & 15 & & \\
Aspect $\left(^{\circ}\right)$ & 45 & 45 & 225 & & & & & & & & 250 & 204 & 280 & 289 & 253 & 174 & & 28 \\
Total vegetation cover (\%) & 30 & 30 & 5 & 30 & 25 & 30 & 15 & 65 & 55 & 25 & 15 & 70 & 12 & 10 & 70 & 10 & 45 & 15 & 25 & 30 & 60
\end{tabular}

D. s. of ass. Erysimo cretacei-Festucetum cretacei

Erysimum ucranicum

Melilotus officinalis

$\begin{array}{llllll}1 & 1 & 1 & 1 & 1 & 1 \\ 1 & 1 & \cdot & 1 & \cdot & 1 \\ 1 & 2 & 1 & 2 & 1 & 2\end{array}$

Festuca cretaced

D. s. of ass. Lepidio meyeri-Scrophularietum cretacei

Lepidium meyeri

Hedysarum cretaceum

Galium octonarium

Artemisia absinthium

Poa compressa

Thesium arvense

D. s. of alliance Artemisio hololeucae-Hyssopion cretacei

Scrophularia cretacea

Hyssopus officinalis

Matthiola fragrans

Linaria cretacea

D. s. of alliance Euphorbio cretophilae-Thymion cretacei Artemisia salsoloides

D. s. of alliance Centaureo carbonatae-Koelerion talievii Stipa capillata

Campanula sibirica

D. s. of order Thymo cretacei-Hyssopetalia cretacei

Pimpinella tragium

Gypsophila oligosperma

$\begin{array}{lllllllllllllllllllll}2 & 2 & 2 & . & . & 2 & 2 & . & . & 2 & . & . & 2 & 1 & 1 & 1 & 1 & 2 & 2 & . & . \\ 4 & 2 & 1 & 2 & 4 & 4 & 4 & . & . & 4 & 2 & 1 & 3 & 2 & 1 & 2 & 1 & 2 & 3 & 2 & 1 \\ . & . & . & . & . & . & . & . & . & . & . & . & . & 1 & . & 1 & . & 1 & . & . & .\end{array}$

Cephalaria uralensis

Reseda lutea

Polygala cretacea

Astragalus albicaulis

Taraxacum serotinum

Orthantha lutea

D. s. of class Festuco-Brometea

Festuca valesiaca

Hieracium virosum

Medicago falcata

Achillea nobilis

Another species

Artemisia nutans

Euphorbia petrophila

Koeleria pyramidata

Helichrysum arenarium

Echinops ritro

Melica transsilvanica

Poa angustifolia 


\begin{tabular}{lcccccccccccccccccccccc}
\hline Relevé number & 1 & 2 & 3 & 4 & 5 & 6 & 7 & 8 & 9 & 10 & 11 & 21 & 12 & 13 & 22 & 14 & 23 & 15 & 18 & 19 & 24 \\
\hline $\begin{array}{l}\text { Securigera varia } \\
\text { Cichorium intybus }\end{array}$ &. &. &. & 1 &. &. &. & 2 & 1 &. &. &. &. &. &. &. &. &. &. &. &. \\
Poa bulbosa &. &. &. &. &. & 1 &. &. & 1 &. &. &. &. &. &. &. &. &. &. &. &. \\
\hline
\end{tabular}

Localities: 1 - right bank of the Don River, Rostov reg.; Russia; 07.2009 (M. Sereda)

Localities: 2 - right bank of the Don River, Rostov reg.; Russia; 07.2009 (M. Sereda)

Localities: 3 - right bank of the Don River, Rostov reg.; Russia; 07.2009 (M. Sereda)

Localities: 4 - Verkhnedonskoy district, 3 km west of the village Demidovskiy, Chayka, Rostov reg.; Russia; 27.06.2009

(O. Demina)

Localities: 5 - Verkhnedonskoy district, vicinity of village Stogovskoy, Rostov reg.; Russia; 02.06.2008 (O. Demina)

Localities: 6 - Verkhnedonskoy district, vicinity of village Stogovskoy, Belogorskoe hole, Rostov reg.; Russia; 04.06.2009

(O. Demina)

Localities: 7 - Verkhnedonskoy district, $1 \mathrm{~km}$ north of the village Stogovskoy, Rostov reg.; Russia; 05.06.2009 (O. Demina)

Localities: 8 -Verkhnedonskoy district, 3 km west from of the Demidovskiy, Chayka, Rostov reg.; Russia; 27.06.2009

(O. Demina)

Localities: 9 - Chertkovskiy district, $2 \mathrm{~km}$ north of the village Mankovo-Kalitvenskoe, Rostov reg.; Russia; 07.06.2009

(O. Demina)

Localities: 10 - Verkhnedonskoy district, vicinity of village Stogovskoy, Belogorskoe hole, Rostov reg.; Russia; 04.06.2009 (O. Demina)

Localities: 11 - Zatonsky, Rostov reg.; Russia; 25.08.2014 (O. Demina)

Localities: 21 - Sholokhovskiy district, vicinities of village Zatonskiy, Rostov reg.; Russia; 06.07.2009 (O. Demina)

Localities: 12 - Zatonsky, Rostov reg.; Russia; 25.08.2014 (O. Demina)

Localities: 13 - Zatonsky, Rostov reg.; Russia; 25.08.2014 (O. Demina)

Localities: 22 - Sholokhovskiy district, vicinities of village Zatonskiy, Rostov reg.; Russia; 06.07.2009 (O. Demina)

Localities: 14 - Zatonsky, Rostov reg.; Russia; 25.08.2014 (O. Demina)

Localities: 23 - Sholokhovskiy district, vicinities of village Zatonskiy, Rostov reg.; Russia; 06.07.2009 (O. Demina)

Localities: 15 - Zatonsky, Rostov reg.; Russia; 25.08.2014 (O. Demina)

Localities: 18 - Zatonsky, Rostov reg.; Russia; 25.08.2014 (O. Demina)

Localities: 19 - Zatonsky, Rostov reg.; Russia; 25.08.2014 (O. Demina)

Localities: 24 - Sholokhovskiy district, vicinities of village Zatonskiy, Rostov reg.; Russia; 06.07.2009 (O. Demina) 
Appendix 1b: Characteristic table of syntaxa of the alliance Euphorbio cretophilae-Thymion cretacei.

Priloga 1b: Tabela sintaksonov zveze Euphorbio cretophilae-Thymion cretacei.

\begin{tabular}{lcccccccccccccccccccc}
\hline Consecutive number & 1 & 2 & 3 & 4 & 5 & 6 & 7 & 8 & 9 & 10 & 11 & 12 & 13 & 14 & 15 & 16 & 17 & 18 & 19 & 20 \\
\hline Total vegetation cover (\%) & 20 & 70 & 80 & 60 & 70 & 60 & 20 & 60 & 31 & 20 & 50 & 60 & 60 & 50 & 50 & 40 & 60 & 60 & 50 & 50 \\
Slope $\left({ }^{\circ}\right)$ & & 30 & 15 & 10 & 20 & 8 & 20 & 5 & & 40 & 40 & 40 & 30 & 30 & 40 & 30 & 30 & 45 & 40 & 40 \\
Aspect $\left({ }^{\circ}\right)$ & & 135 & 155 & 180 & 225 & 315 & 180 & 135 & & 315 & 180 & 135 & 135 & 135 & 135 & 225 & 135 & 180 & 225 & 180 \\
Species richness & 16 & 24 & 19 & 12 & 18 & 15 & 19 & 20 & 29 & 19 & 19 & 18 & 19 & 17 & 19 & 15 & 19 & 19 & 20 & 19
\end{tabular}

D. s. of ass. Euphorbio cretophilae-Jurinetum brachycephalae

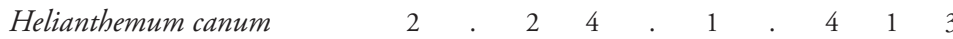

D. s. of ass. Genisto scythicae-Artemisietum salsoloides

Genista scythica

Rhaponticoides taliewii

Leontodon biscutellifolius

Linum tenuifolium

Odontarrhena tortuosa

Linaria vulgaris

Diplotaxis cretacea

Polygala comosa

Linum hirsutum

D. s. of alliance Artemisio hololeucae-Hyssopion cretacei

Scrophularia cretacea

Hyssopus cretaceus

Matthiola fragrans

Linaria cretacea

D. s. of alliance Euphorbio cretophilae-Thymion cretacei Jurinea stoechadifolia

Euphorbia petrophila

Artemisia salsoloides

Brassica elongata

D. s. of alliance Centaureo carbonatae-Koelerion talievii

Campanula sibirica

Carex humilis

Stipa pennata

Onosma simplicissima

Koeleria talievii

Psephellus marschallianus

Teucrium polium

D. s. of order Thymo cretacei-Hyssopetalia cretacei

Pimpinella tragium

Gypsophila oligosperma

Cephalaria uralensis

Thymus calcareus

Asperula tephrocarpa

Reseda lutea

Silene supina

Linum pallasianum

Orthantha lutea

Scutellaria supina

Linaria genistifolia 
D. s. of class Festuco-Brometea

Euphorbia seguieriana

Galium ruthenicum

Potentilla recta

Astragalus sulcatus

Adonis volgensis

Another species

Polygala cretacea

Convolvulus lineatus

Thesium arvense

Artemisia hololeuca

Allium inaequale

Hedysarum grandiflorum

Asperula cynanchica

Salvia verticillata

Caragana frutex

Centaurea orientalis

Bupleurum falcatum

Anisantha tectorum

Scabiosa ochroleuca

Koeleria pyramidata

Bromopsis riparia

Salvia nutans

Thalictrum minus

Helichrysum arenarium

Cytisus ruthenicus

Viola ambigua

Echinops ritro

Stipa lessingiana

Erysimum diffusum

Ephedra distachya

Localities: 1 - vil. Zakotnoe, Donetsk reg.; Ukraine; 08.1994 (K. Romashchenko)

Localities: 2 - vil. Nyzhnye, Lugansk reg.; Ukraine; 06.1987 (Ya. Didukh)

Localities: 3 - "Kreidova flora", Donetsk reg.; Ukraine; 08.2016 (O. Chusova)

Localities: 4 - "Kreidova flora", Donetsk reg.; Ukraine; 08.2016 (O. Chusova)

Localities: 5 - "Kreidova flora", Donetsk reg.; Ukraine; 08.2016 (O. Chusova)

Localities: 6 - vil. Kryva Luka, Donetsk reg.; Ukraine; 22.06.1987 (Ya. Didukh)

Localities: 7 - vil. Bogorodychnoe, Donetsk reg.; Ukraine; 08.1994 (K. Romashchenko)

Localities: 8 - vil. Kryva Luka, Donetsk reg.; Ukraine; 22.06.1987 (Ya. Didukh)

Localities: 9 - vil. Bogorodychnoe, Donetsk reg.; Ukraine; 08.1994 (K. Romashchenko)

Localities: 10 - "Kreidova flora”, Donetsk reg.; Ukraine; 28.08.1994 (K. Romashchenko)

Localities: 11 - right bank of Tuzlov River, vil. Lysogorka, Rostov reg.; Russia; 4.06.2002 (M. Sereda)

Localities: 12 - right bank of Tuzlov River, vil. Lysogorka, Rostov reg.; Russia; 4.06.2002 (M. Sereda)

Localities: 13 - right bank of Tuzlov River, vil. Lysogorka, Rostov reg.; Russia; 4.06.2002 (M. Sereda)

Localities: 14 - right bank of Tuzlov River, vil. Lysogorka, Rostov reg.; Russia; 4.06.2002 (M. Sereda)

Localities: 15 - right bank of Tuzlov River, vil. Lysogorka, Rostov reg.; Russia; 4.06.2002 (M. Sereda)

Localities: 16 - right bank of Tuzlov River, vil. Lysogorka, Rostov reg.; Russia; 4.06.2002 (M. Sereda)

Localities: 17 - right bank of Tuzlov River, vil. Lysogorka, Rostov reg.; Russia; 4.06.2002 (M. Sereda)

Localities: 18 - right bank of Tuzlov River, vil. Lysogorka, Rostov reg.; Russia; 4.06.2002 (M. Sereda)

Localities: 19 - right bank of Tuzlov River, vil. Lysogorka, Rostov reg.; Russia; 4.06.2002 (M. Sereda)

Localities: 20 - right bank of Tuzlov River, vil. Lysogorka, Rostov reg.; Russia; 4.06.2002 (M. Sereda) 
Appendix 1c: Characteristic table of syntaxa of the alliance Centaureo carbonatae-Koelerion talievii.

Priloga 1c: Tabela sintaksonov zveze Centaureo carbonatae-Koelerion talievii.

\begin{tabular}{llllllllllllllllllllllllllllllll}
\hline Consecutive number & 1 & 2 & 3 & 4 & 5 & 6 & 7 & 8 & 9 & 10 & 11 & 12 & 13 & 14 & 15 & 16 & 17 & 18 & 19 & 20 & 21 & 22 & 23 & 24 & 25 & 26 & 27 & 28 & 29 & 30
\end{tabular}

Total vegetation cover (\%) $70 \begin{array}{lllllllllllllllllllllllllllll}70 & 60 & 90 & 60 & 65 & 65 & 70 & 50 & 70 & 40 & 60 & 50 & 65 & 65 & 45 & 50 & 60 & 80 & 40 & 15 & 40 & 40 & 60 & 10 & 10 & 60 & 80 & 40\end{array}$

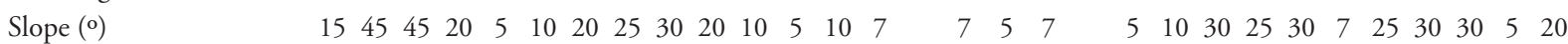

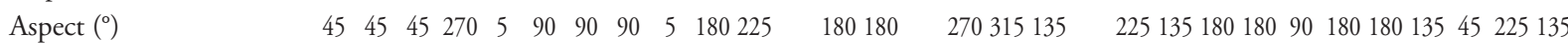

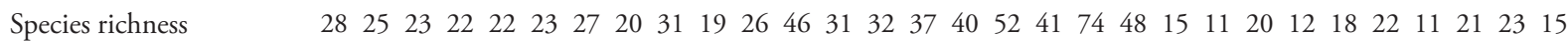

D.s. ass. Pimpinello titanophillae-Artemisietum salsoloides

$\begin{array}{lllllllllll}\text { Artemisia salsoloides } & 5 & 1 & 4 & 1 & 3 & 5 & . & 3 & 4\end{array}$

Polygala cretacea

$\begin{array}{lllllllll}1 & 1 & 1 & \cdot & 1\end{array}$

Linum hirsutum

$12 . \quad 2$

D.s. ass. Hedysaro grandiflori-Psephelletum carbonatae

Allium inaequale

Hedysarum grandiflorum

Thymelaea passerina

Asperula cynanchica

Adonis vernalis

Seseli annuum

Pimpinella saxifraga

Phlomis herba-venti

Salvia verticillata

Aster amellus

Asparagus officinalis

Caragana frutex

Centaurea orientalis

D.s. ass. Androsacio koso-poljanskii-Caricetum humilis

Androsace villosa

D. s. of alliance Artemisio hololeucae-Hyssopion cretacei

Artemisia hololeuca

Scrophularia cretacea

Hyssopus cretaceus

Matthiola fragrans

D. s. of alliance Euphorbio cretophilae-Thymion cretacei

Brassica elongata

D. s. of alliance Centaureo carbonatae-Koelerion talievii

Stipa capillata

Campanula sibirica

Polygala sibirica

Carex humilis

Stipa pennata

Onosma simplicissima

Koeleria talievii

Psephellus marschallianus

Teucrium polium

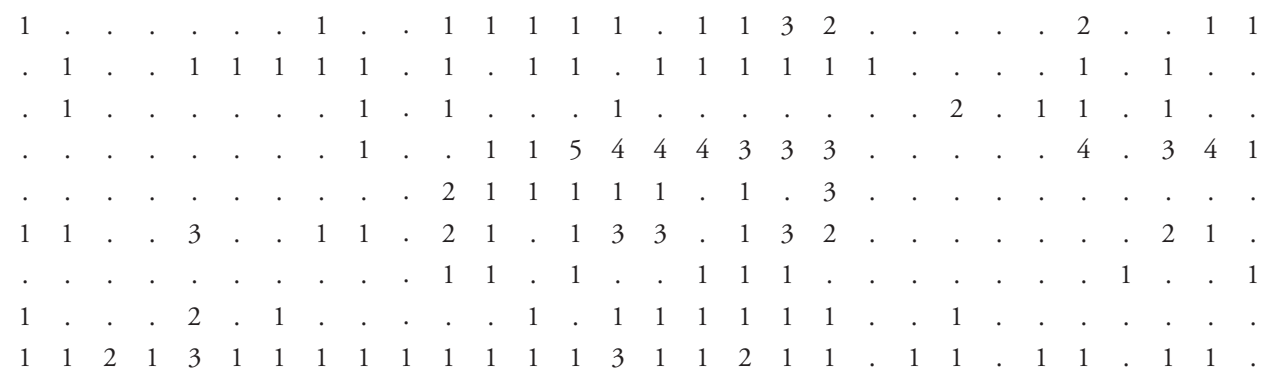

D. s. of order Thymo cretacei-Hyssopetalia cretacei

Pimpinella tragium

Gypsophila oligosperma

$\begin{array}{lllllllllllllllllllllllllllllll}. & 3 & 1 & . & . & 1 & 4 & 1 & 1 & 1 & 1 & 1 & 1 & 1 & . & . & 1 & . & . & . & 2 & 1 & 1 & 1 & 2 & 1 & 2 & 2 & . & 1 \\ 2 & 3 & 1 & 1 & 1 & 1 & . & . & 1 & . & 1 & . & 1 & 1 & 1 & 1 & 1 & 4 & 1 & 1 & . & . & 1 & 1 & 1 & 1 & 1 & . & 1 & . \\ 1 & . & 1 & . & 1 & . & . & . & 1 & 1 & 2 & . & 2 & 1 & . & . & 1 & 1 & 1 & . & . & 1 & . & . & . & . & . & . & . & . \\ 1 & 4 & 1 & 1 & . & 3 & 3 & 3 & 3 & . & 3 & 1 & 4 & 1 & 3 & 2 & 1 & 1 & 3 & . & 3 & 3 & 4 & 4 & 1 & 1 & 2 & 3 & . & 1 \\ 1 & 1 & 1 & . & 2 & 1 & 2 & 3 & 1 & 1 & . & 1 & . & . & . & . & 1 & . & . & . & 2 & . & 2 & 1 & . & 1 & 1 & . & . & . \\ . & 1 & . & . & . & . & . & 1 & . & . & 1 & 1 & 1 & . & 3 & . & 1 & 1 & 1 & . & . & 1 & . & 1 & . & . & . & . & . & . \\ .\end{array}$

Cephalaria uralensis

Thymus calcareus

Asperula tephrocarpa

Reseda lutea 
Localities: 1 - vil. Baranikivka, Lugansk reg.; Ukraine; 07.1987 (Ya. Didukh)

Localities: 2 - vil. Baranikivka, Lugansk reg.; Ukraine; 07.1987 (Ya. Didukh)

Localities: 3 - vil. Baranikivka, Lugansk reg.; Ukraine; 07.1987 (Ya. Didukh)

Localities: 4-5 km South of the Bilovodsk, Lugansk reg.; Ukraine; 07.1987 (Ya. Didukh)

Localities: 5 - vil. Nyzhnya Duvanka, Lugansk reg.; Ukraine; 13.06.2013 (O. Chusova)

Localities: 6 - right bank of Derkul River, Lugansk reg.; Ukraine; 07.1987 (Ya. Didukh)

Localities: 7 - right bank of Kamyshna River, Lugansk reg.; Ukraine; 07.1987 (Ya. Didukh)

Localities: 8 - right bank of Kobylka River, Lugansk reg.; Ukraine; 06.1987 (Ya. Didukh)

Localities: 9 -vil. Stril'tsivka, Milovs'kyy district; Lugansk reg.; Ukraine; 07.1987 (Ya. Didukh) 
Localities: 10 - 5 km South of the Bilovodsk, Lugansk reg.; Ukraine; 07.1987 (Ya. Didukh)

Localities: 11 - Oskol River, Belogorie Reserve, Belgorod reg.; Russia; 4.08.2012 (E. Averinova)

Localities: 12 - border between Rossoshinsky and Kantemirovsky districts, Voronezh reg.; Russia; 1.08.1983 (A. Grigorevskaya*)

Localities: 13 - vil Makeshkino, Belgorod reg.; Russia; 1.08.2012 (E. Averinova)

Localities: 14 - vil Makeshkino, Belgorod reg.; Russia; 1.08.2012 (E. Averinova)

Localities: $15-0.5 \mathrm{~km}$ north-west of the Veidelevka, Belgorod reg.; Russia; 24.08 .2012 (E. Averinova)

Localities: 16 - Oskol River, Belogorie Reserve, Belgorod reg.; Russia; 3.08.2012 (E. Averinova)

Localities: 17 - $1 \mathrm{~km}$ south-east of the Roven'ki Belgorod reg.; Russia; 6.08.2011 (E. Averinova)

Localities: 18 - 1 km west of the Berezhnyi Farm, Belgorod reg.; Russia; 9.08.2011 (E. Averinova)

Localities: 19 - Oskol River, Belogorie Reserve, Belgorod reg.; Russia; 25.08. 1983 (A. Grigorevskaya*)

Localities: 20 - Oskol River, Belogorie Reserve, Belgorod reg.; Russia; 4.08.2012 (E. Averinova)

Localities: 21 - vil. Kamyanka, Kharkiv reg.; Ukraine; 27.05. 2012 (O. Bezrodnova)

Localities: 22 - vil. Dvorichna, Kharkiv reg.; Ukraine; 26.05. 2012 (O. Bezrodnova)

Localities: 23 - Rogan', Kharkiv reg.; Ukraine; 14.06.1996 (Ya. Didukh)

Localities: 24 - Rogan', Kharkiv reg.; Ukraine; 14.06.1996 (Ya. Didukh)

Localities: 25 - vil. Yaichne, Kharkiv reg.; Ukraine; 06.1996 (Ya. Didukh)

Localities: 26 - south of the Rogan', Kharkiv reg.; Ukraine; 05.1995 (Ya. Didukh)

Localities: 27 - vil. Staromelovoe, Kursk reg.; Russia; 2.08. 2005 (A. Poluyanov)

Localities: 28 - vil. Kamyanka, Kharkiv reg.; Ukraine; 27.05. 2012 (O. Bezrodnova)

Localities: 29 - Pechenigy, Kharkiv reg.; Ukraine; 15.06.1996 (Ya. Didukh)

Localities: 30 - vil. Kamyanka, Kharkiv reg.; Ukraine; 21.08. 2012 (O. Bezrodnova)

* - relevés were taken from Averinova (2014).

Appendix 1c (extension): Characteristic table of syntaxa of the alliance Centaureo carbonatae-Koelerion talievii.

Priloga 1c (nadaljevanje): Tabela sintaksonov zveze Centaureo carbonatae-Koelerion talievii.

Consecutive number

Total vegetation cover $(\%)$

Slope $\left(^{\circ}\right)$

Aspect $\left({ }^{\circ}\right)$

Species richness $\begin{array}{llllllllllllllllllllllllllllll}21 & 22 & 23 & 24 & 25 & 26 & 27 & 28 & 29 & 30 & 31 & 32 & 33 & 34 & 35 & 36 & 37 & 38 & 39 & 40 & 41 & 42 & 43 & 44 & 45 & 46 & 47 & 48 & 49 & 50\end{array}$

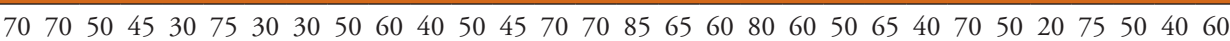

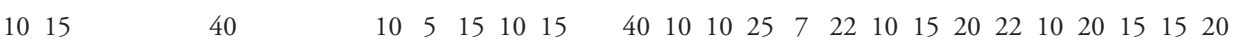
$\begin{array}{llllllllllllllll}70 & 45 & 180 & 90 & 5 & 90 & 90 & 5 & 5 & 270 & 5 & 18045 & 45 & 135225 & 45180180180180180180\end{array}$ $\begin{array}{llllllllllllllllllllllllllllll}17 & 25 & 31 & 31 & 25 & 24 & 18 & 14 & 26 & 21 & 30 & 22 & 26 & 25 & 36 & 33 & 27 & 27 & 40 & 25 & 24 & 31 & 30 & 28 & 26 & 27 & 29 & 23 & 27 & 29\end{array}$

D. s. of ass. Bupleuro falcatae-Stipetum capillatae

\begin{tabular}{|c|c|c|c|c|c|c|c|c|c|c|c|c|c|c|c|c|c|c|c|c|c|c|c|c|c|c|c|c|c|c|}
\hline Bupleurum falcatum & 1 & 1 & 1 & 1 & 1 & 1 & 1 & 1 & 1 & $\cdot$ & . & 1 & 1 & $\cdot$ & $\cdot$ & . & . & 1 & . & . & 1 & & 1 & 1 & & & & & 1 & 1 \\
\hline Anisantha tectorum & . & . & 1 & 1 & ${ }^{\circ}$ & $\cdot$ & 1 & . & $\cdot$ & ${ }^{\circ}$ & . & . & . & . & . & ${ }^{\circ}$ & . & 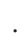 & . & . & . & & ${ }^{\circ}$ & & & & & & & . \\
\hline Scabiosa ochroleuca & 1 & 1 & 1 & 1 & 1 & . & 1 & 1 & 1 & 3 & . & ${ }^{\circ}$ & 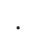 & 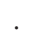 & 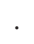 & 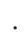 & . & 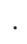 & . & 2 & . & & & & & & & & 1 & 1 \\
\hline \multicolumn{31}{|c|}{ D. s. of ass. Gypsophilo oligospermae-Campanuletum sibiricae } \\
\hline Carex pediformis & . & . & . & . & 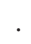 & . & . & . & . & . & 3 & 3 & 3 & 2 & . & . & . & . & . & . & . & & & & & & & & & . \\
\hline Koeleria pyramidata & . & . & . & . & . & $\cdot$ & . & . & . & 1 & 2 & 1 & 2 & 2 & 1 & 1 & . & . & . & 3 & . & & & . & & & & & & . \\
\hline Nonea pulla & . & . & . & . & $\cdot$ & . & . & . & . & . & . & . & 1 & 1 & . & $\cdot$ & $\cdot$ & 1 & . & 2 & . & & & & & & & & . & . \\
\hline Bromopsis riparia & . & . & . & . & $\cdot$ & . & . & . & . & 2 & 2 & . & 3 & 4 & 1 & 2 & . & 1 & 1 & . & 1 & $\cdot$ & & & & & & & . & · \\
\hline Salvia nutans & . & . & 1 & 1 & 1 & 1 & 1 & 2 & 1 & . & 1 & . & 3 & 2 & 1 & 2 & 1 & 1 & 3 & 3 & . & & & & & & & & . & . \\
\hline Galatella villosa & . & . & . & . & . & . & . & . & . & . & 4 & . & 1 & 1 & $\cdot$ & 4 & . & . & 1 & 2 & & & & & & & & & & \\
\hline Linum hirsutum & 1 & 1 & . & 1 & . & . & . & . & . & . & 1 & 2 & 2 & 2 & 1 & & 2 & . & & & & & & & & & & & & \\
\hline
\end{tabular}

D. s. of ass. Carici humilis-Thymetum calcarei

Androsace villosa

Astragalus austriacus

Thalictrum minus

Oxytropis pilosa

Jurinea arachnoidea

Viola rupestris

Helianthemum nummularium

Asperula tinctoria

Anthericum ramosum

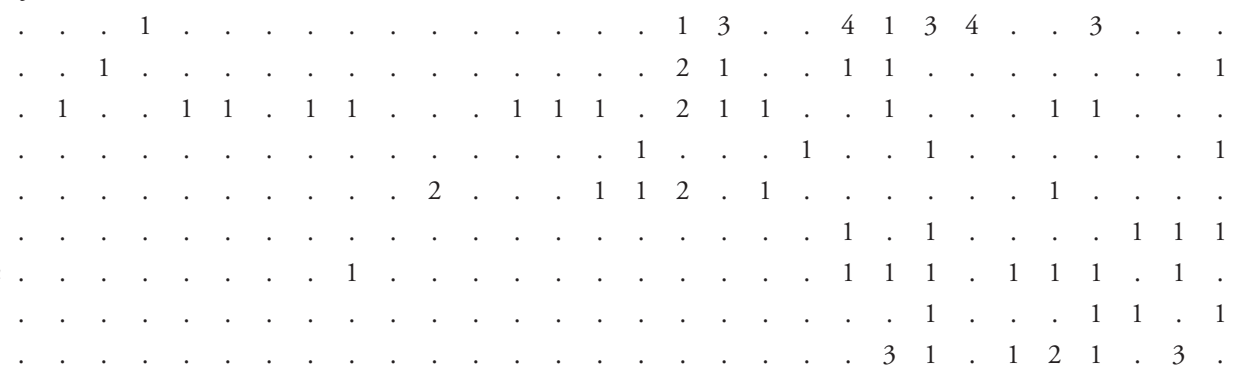


Linum perenne

Vincetoxicum hirundinaria

Euphrasia pectinata

D. s. of alliance Artemisio hololeucae-Hyssopion cretacei

Artemisia hololeuca

D. s. of alliance Euphorbio cretophilae-Thymion cretacei

Brassica elongata

Jurinea stoechadifolia

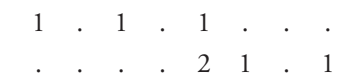

Euphorbia petrophila

D. s. of allliance Centaureo carbonatae-Koelerion talievii

Stipa capillata

Campanula sibirica

Polygala sibirica

Carex humilis

Stipa pennata

Onosma simplicissima

Koeleria talievii

Psephellus marschallianus

Teucrium polium

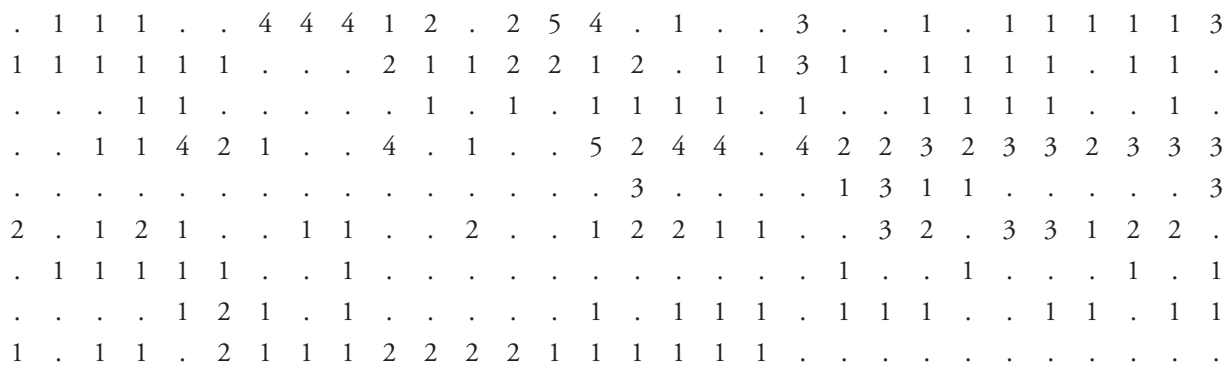

D. s. of order Thymo cretacei-Hyssopetalia cretacei

\begin{tabular}{|c|c|c|c|c|c|c|c|c|c|c|c|c|c|c|c|c|c|c|c|c|c|c|c|c|c|c|c|c|c|}
\hline Pimpinella tragium & 1 & 1 & 1 & 1 & & 1 & & & & & & 2 & & & 1 & 1 & & 1 & 1 & & 1 & & 1 & 1 & & & 1 & & \\
\hline Gypsophila oligosperma & 1 & 2 & 1 & 1 & 1 & . & . & & & 2 & & 1 & . & 1 & & & . & . & . & 1 & 1 & & 1 & 1 & 1 & & 1 & 1 & 1 \\
\hline Cephalaria uralensis & . & . & . & . & . & . & 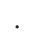 & 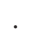 & & . & & 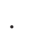 & . & . & 1 & & . & . & . & . & . & & . & . & . & & . & . & 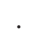 \\
\hline Thymus calcareus & 4 & 4 & 3 & 4 & 2 & 1 & . & . & . & 2 & & 4 & . & . & 1 & . & 1 & 1 & . & . & 1 & 1 & 2 & 2 & 3 & 1 & 4 & 3 & 1 \\
\hline Asperula tephrocarpa & 1 & 1 & 1 & 1 & 1 & 1 & 1 & 1 & 1 & 1 & 2 & 1 & 1 & 1 & 1 & 1 & 1 & 1 & 1 & . & . & & . & . & & & . & . & 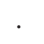 \\
\hline Reseda lutea & 1 & 1 & 1 & 1 & . & $\cdot$ & . & $\cdot$ & . & 2 & 2 & 3 & 1 & 1 & & 1 & . & . & 1 & 2 & . & & $\cdot$ & . & & & 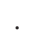 & $\cdot$ & . \\
\hline Silene supina & . & . & 1 & 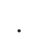 & . & . & . & . & . & . & 2 & 1 & 1 & 1 & $\cdot$ & . & . & . & . & 1 & . & . & . & . & & & . & $\cdot$ & r. \\
\hline Linum pallasianum & 1 & 1 & 2 & 2 & 1 & . & . & . & . & 2 & 2 & 1 & 3 & 1 & 1 & 2 & . & 1 & 1 & . & . & . & 1 & . & . & & 1 & . & . \\
\hline Polygala cretacea & 1 & 1 & . & . & . & . & . & . & . & . & . & . & . & $\cdot$ & 1 & . & 2 & $\cdot$ & . & . & . & 1 & . & . & . & & . & $\cdot$ & . \\
\hline Astragalus albicaulis & 1 & 1 & 1 & 1 & . & . & . & . & . & . & . & . & . & . & • & . & . & . & . & . & . & & . & . & 1 & & & . & 1 \\
\hline Genista tinctoria & . & . & . & . & . & . & . & . & $\cdot$ & . & 2 & . & 1 & 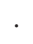 & . & 3 & . & . & . & 3 & . & & . & . & & & . & . & . \\
\hline Orthantha lutea & 1 & 1 & 1 & 1 & 1 & 1 & 1 & . & 1 & . & 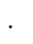 & . & . & . & . & $\cdot$ & . & . & . & . & $\cdot$ & & . & . & $\cdot$ & & . & $\cdot$ & . \\
\hline Helianthemum canum & . & 1 & 1 & 1 & . & . & . & . & . & . & 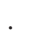 & 4 & . & . & . & . & . & . & . & . & . & & . & . & & & . & . & . \\
\hline Odontarrhena tortuosa & . & . & . & . & 1 & . & . & . & . & . & 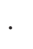 & 3 & . & . & 1 & . & . & . & . & . & . & & . & . & & & &. & . \\
\hline Diplotaxis cretacea & . & . & . & . & . & 1 & 1 & 1 & . & 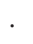 & & . & . & . & . & . & 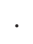 & . & . & . & . & & 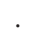 & 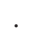 & & & & . & . \\
\hline Artemisia salsoloides & . & . & . & . & . & $\cdot$ & . & . & . & 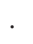 & . & . & . & & 2 & . & . & . & 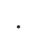 & . & . & & . & . & & & . & . & 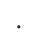 \\
\hline Plantago maritima & . & . & & & . & & . & . & & . & & . & . & & & 2 & & . & & . & & & & & & & & & \\
\hline
\end{tabular}

\title{
D. s. of class Festuco-Brometea
}

$\begin{array}{lllllllllllllllllllllllllllllllllllll}\text { Festuca valesiaca } & . & 1 & 2 & . & . & 1 & . & . & 1 & . & 4 & 1 & 4 & 3 & 1 & 2 & 3 & 1 & 1 & 3 & . & 2 & 1 & 1 & . & . & 2 & 1 & 1 & 1\end{array}$ Euphorbia seguieriana

Hieracium virosum

Euphorbia nicaeensis

Stachys recta

Medicago falcata

Potentilla humifusa

Rhaponticoides ruthenica

Onobrychis arenaria

Plantago media

Achillea setacea

Potentilla incana

Potentilla recta

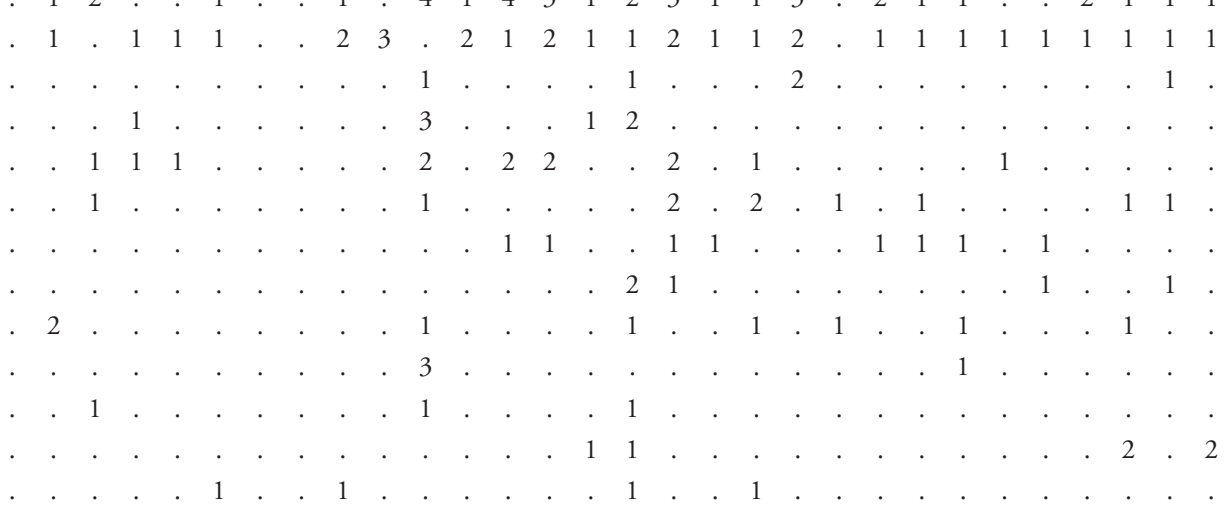


Localities: 21 - right bank of Oskol River, vil. Znamenka, Kharkiv reg.; Ukraine; 21.08.1994 (K. Romashchenko) Localities: 22 - right bank of Oskol River, vil. Znamenka, Kharkiv reg.; Ukraine; 21.08.1994 (K. Romashchenko) Localities: 23 - right bank of Oskol River, vil. Znamenka, Kharkiv reg.; Ukraine; 9.08.1994 (K. Romashchenko) Localities: 24 - right bank of Oskol River, vil. Topolya, Kharkiv reg.; Ukraine; 08.1994 (K. Romashchenko) Localities: 25 - right bank of Oskol River, vil. Nyzhnya Melnitsa, Kharkiv reg.; Ukraine; 08.1994 (K. Romashchenko) Localities: 26 - vil. Lavrentievka, Donetsk reg.; Ukraine; 1.08 .1994 (K. Romashchenko) Localities: 27 - vil. Kryva Luka, Donetsk reg.; Ukraine; 26.08.1994 (K. Romashchenko) Localities: 28 - vil. Kryva Luka, Donetsk reg.; Ukraine; 26.08.1994 (K. Romashchenko) 
Localities: 29 - vil. Kryva Luka, Donetsk reg.; Ukraine; 26.08.1994 (K. Romashchenko)

Localities: 30 - vil. Preobrazhenne, Lugansk reg.; Ukraine; 11.07.2013 (O. Chusova)

Localities: 31 - vil. Preobrazhenne, Lugansk reg.; Ukraine; 14.06.2013 (O. Chusova)

Localities: 32 - vil. Fomovka, Lugansk reg.; Ukraine; 14.06.2013 (Ya. Didukh)

Localities: 33 - vil. Fomovka, Lugansk reg.; Ukraine; 14.06.2013 (Ya. Didukh)

Localities: 34 - vil. Fomovka, Lugansk reg.; Ukraine; 14.06.2013 (Ya. Didukh)

Localities: 35 - 5 km South of the Bilovodsk, Lugansk reg.; Ukraine; 06.1987 (Ya. Didukh)

Localities: 36 - vil. Preobrazhenne, Lugansk reg.; Ukraine; 14.06.2013 (O. Chusova)

Localities: 37 - right bank of Volchya River, vil. Ohrymivka, Kharkiv reg.; Ukraine; 06.1995 (Ya. Didukh)

Localities: 38 - right bank of Oskol River, vil. Dvorichna, Kharkiv reg.; Ukraine; 06.1995 (Ya. Didukh)

Localities: 39 - vil. Kalynivka, Lugansk reg.; Ukraine; 06.1987 (Ya. Didukh)

Localities: 40 - vil. Preobrazhenne, Lugansk reg.; Ukraine; 14.06.2013 (O. Chusova)

Localities: 41 - 1 km South of vil. Aleksandrovka, Kursk reg.; Russia; 4.09. 2005 (A. Poluyanov)

Localities: 42 - right bank of Oskol River, 1 km North of vil. Kun'e, Kursk reg.; Russia; 11.05. 2005 (A. Poluyanov)

Localities: 43 - 6 km South of vil. Novomelovoe, Kursk reg.; Russia; 1.08. 2005 (A. Poluyanov)

Localities: $44-1$ km South of vil. Aleksandrovka, Kursk reg.; Russia; 4.09. 2005 (A. Poluyanov)

Localities: 45 - 0.5 km East of vil. Midlle Apochka, Kursk reg.; Russia; 4.09.2005 (A. Poluyanov)

Localities: 46 - 2 km North of vil. Midlle Apochka, Kursk reg.; Russia; 5.09.2005 (A. Poluyanov)

Localities: 47 - 6 km South of vil. Novomelovoe, Kursk reg.; Russia; 1.08.2005 (A. Poluyanov)

Localities: 48 - 1 km North of vil. Nizhnedorozhnoe, Kursk reg.; Russia; 4.08.2005 (A. Poluyanov)

Localities: $49-2$ km North of vil. Midlle Apochka, Kursk reg.; Russia; 5.09.2005 (A. Poluyanov)

Localities: 50 - left bank of Bystrik River, 1 km East of vil. Beketovo, Kursk reg.; Russia; 27.05.2006 (A. Poluyanov) 